\title{
Modeling a Neurexin-3 $\alpha$ Human Mutation in Mouse Neurons Identifies a Novel Role in the Regulation of Transsynaptic Signaling and Neurotransmitter Release at Excitatory Synapses
}

\author{
논 Susana Restrepo, Nora J. Langer, Kylan A. Nelson, and 지ason Aoto \\ Department of Pharmacology, University of Colorado Denver School of Medicine, Aurora, Colorado 80045
}

Presynaptic $\alpha$-neurexins are highly expressed and more frequently linked to neuropsychiatric and neurodevelopmental disorders than $\beta$-neurexins. However, how extracellular sequences specific to $\alpha$-neurexins enable synaptic transmission is poorly understood. We identified a mutation in an extracellular region of neurexin-3 $\alpha$ (A687T), located in a region conserved among $\alpha$-neurexins and throughout vertebrate evolution, in a patient diagnosed with profound intellectual disability and epilepsy. We systematically interrogated this mutation using a knockdown-replacement approach, and discovered that the A687T mutation enhanced presynaptic morphology and increased two critical presynaptic parameters: (1) presynaptic release probability, and (2) the size of the readily releasable pool exclusively at excitatory synapses in mixed sex primary mouse hippocampal cultures. Introduction of the mutation in vivo and subsequent analysis in ex vivo brain slices made from male and female mice revealed a significant increase in excitatory presynaptic neurotransmission that occluded presynaptic but not postsynaptic LTP. Mechanistically, neurexin-3 $\alpha^{\mathrm{A} 687 \mathrm{~T}}$ enhanced binding to LRRTM2 without altering binding to postsynaptic neuroligin- 1 . Thus, neurexin- $3 \alpha^{\mathrm{A} 687 \mathrm{~T}}$ unexpectedly produced the first neurexin presynaptic gain-offunction phenotype and revealed unanticipated novel insights into how $\alpha$-neurexin extracellular sequences govern both transsynaptic adhesion and presynaptic neurotransmitter release.

Key words: $\alpha$ neurexin; human mutation; neurexin; presynaptic release

\section{Significance Statement}

Despite decades of scientific scrutiny, how precise $\alpha$-neurexin extracellular sequences control synapse function remains enigmatic. One largely unpursued avenue to identify the role of precise extracellular sequences is the interrogation of naturally occurring missense mutations. Here, we identified a neurexin- $3 \alpha$ missense mutation in a compound heterozygous patient diagnosed with profound intellectual disability and epilepsy and systematically interrogated this mutation. Using in vitro and in vivo molecular replacement, electrophysiology, electron microscopy, and structure-function analyses, we reveal a novel role for neurexin- $3 \alpha$, unanticipated based on $\alpha$-neurexin knock-out models, in controlling presynaptic morphology and neurotransmitter release at excitatory synapses. Our findings represent the first neurexin gain-of-function phenotype and provide new fundamentally important insight into the synaptic biology of $\alpha$-neurexins.

\section{Introduction}

Neurexins (Nrxns) are a family of essential presynaptic cell adhesion molecules that are thought to play pleiotropic roles in syn- apse formation, maintenance, and plasticity in a brain region, cell-type-, and synapse-specific manner, however, the exact roles each neurexin performs at the synapse are still poorly defined (Aoto et al., 2015; Südhof, 2017; Dai et al., 2019). Nrxns are

discussions, Dr. Samantha Schwartz for MATLAB programming, Dr. Joshua Black for q-PCR use, Dr. Jennifer Bourne in the Electron Microscopy Center at the University of Colorado School of Medicine, Dr. Scott Demarest for the COMIRB protocol, and Thomas Südhof (Stanford University) for the NL2 and LRRTM2 plasmids.

The authors declare no competing financial interests.

Correspondence should be addressed to Jason Aoto at jason.aoto@ucdenver.edu.

https://doi.org/10.1523/JNEUROSCI.1261-19.2019

Copyright $\odot 2019$ the authors 
A

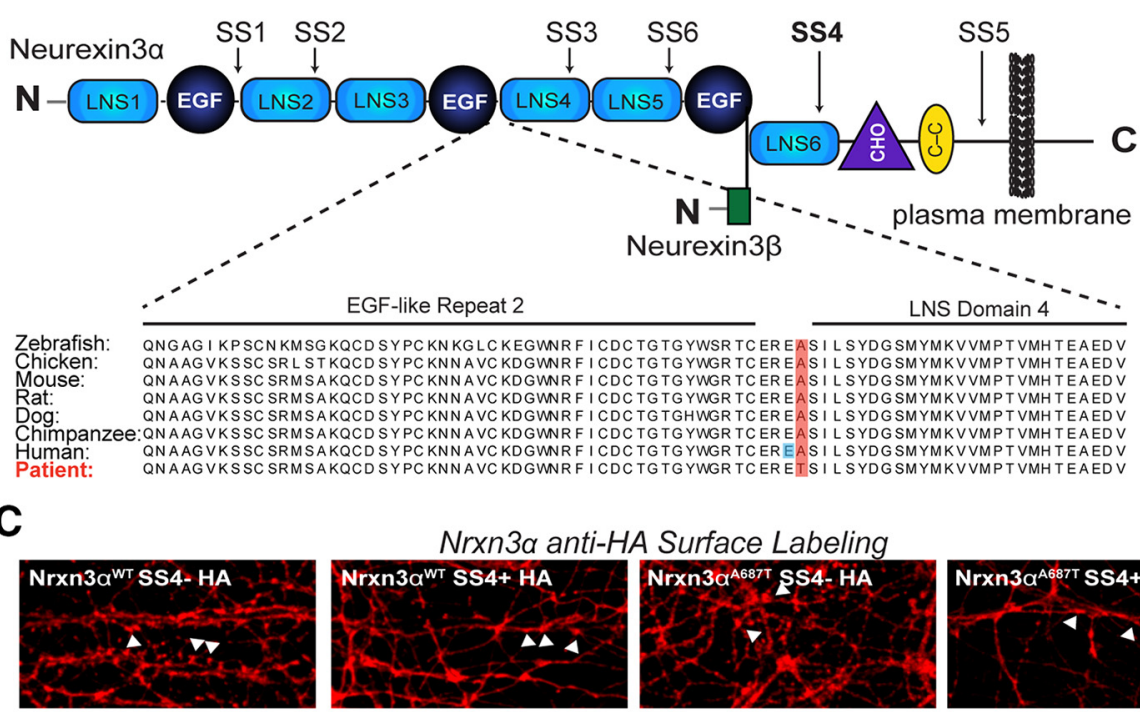

C
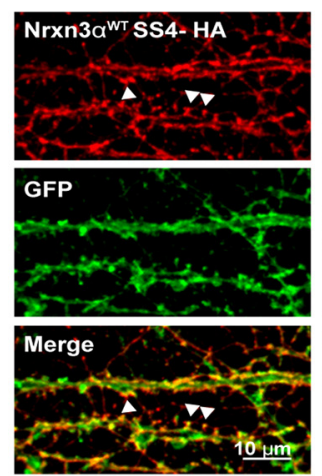

Location of Neurexin-3 $3 \alpha^{A 687 T}$ Mutation

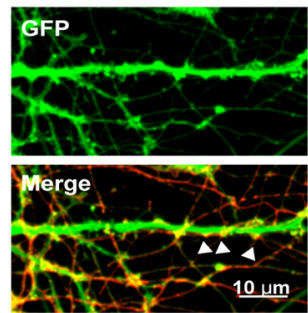

Nrxn3 $\alpha$ anti-HA Surface Labeling
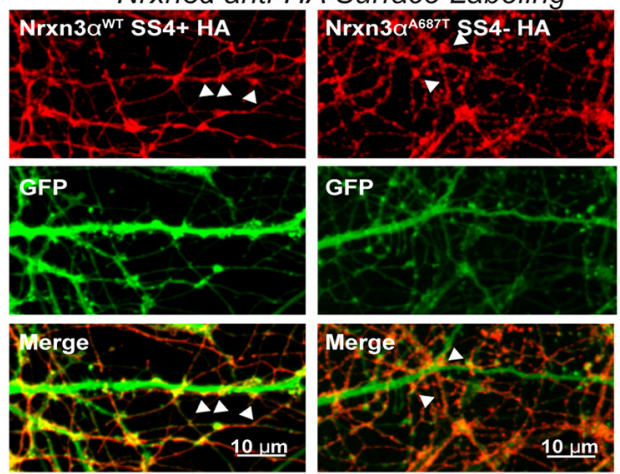

B
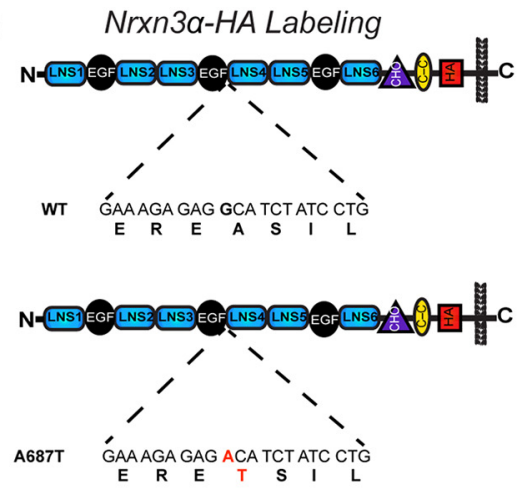

Figure 1. The A687T mutation does not alter surface trafficking of Nrxn3 $\alpha$. A, Location of the A687T mutation in Nrxn3 $\alpha$. Mutations in this region in Nrxn1 $\alpha$ are highlighted in blue and imposed onto the Nrxn $3 \alpha$ protein sequence. $\boldsymbol{B}$, Diagram depicting the $\mathrm{G}>$ A mutation in the Nrxn $3 \alpha$ gene and the relative location of HA-epitope tag. $\boldsymbol{C}$, Representative images and quantification of live surface labeled HA-tagged SS4 isoforms of Nrxn3 $\alpha^{\text {WT }}$ or Nrxn3 $\alpha^{\text {A687T }}$. Overexpressed HA-Nrxn3 $\alpha$ SS4 isoforms localize to axons. White arrowheads point to presence of Nrxn3 $\alpha$ opposed to dendritic spines. Data shown represent the mean \pm SEM of the number of experiments. Number presented in bars represent cells/number of experiments. Statistical significance was determined by one-way ANOVA, multiple comparisons.

encoded by three evolutionarily conserved genes (Nrxn1-3) that give rise to longer $\alpha$ and shorter $\beta$ neurexin gene products from two independent promoters (Südhof, 2017). $\alpha$-Nrxns and $\beta$-Nrxns share transmembrane and cytoplasmic sequences, which promote presynaptic function by interacting with synaptotagmin-1, CASK, Mint-1, and Veli (Reissner et al., 2013), but differ in the length and complexity of their extracellular sequences. $\alpha$-Nrxn extracellular sequences are comprised of six laminin-neurexin-sex hormone (LNS1-6) domains interspersed with three EGF-like repeats (EGF1-3). $\beta$-Nrxns are essentially truncated $\alpha$-Nrxns that contain only LNS6 (Fig. $1 A$ ). The extracellular region of neurexins is subject to extensive alternative splicing. Although poorly understood, alternative splicing is thought to govern neurotransmission by modulating transsynaptic interactions with postsynaptic ligands.

$\alpha$-Nrxns are alternatively spliced at six conserved sites (SS1-6) that generate over 1000 splice isoforms (Fig. 1A; Ullrich et al., 1995), and of the six conserved splice sites, SS4 has received the most scrutiny. Splice-site 4 is located in LNS6 and is thus shared by $\alpha$ - and $\beta$-Nrxns. The inclusion or exclusion of the alternative SS4 exon controls transsynaptic interactions with almost all known neurexin ligands (Südhof, 2017). Although largely untested, Nrxn $1 \alpha$ crystal structures suggest that $\alpha$-Nrxns function as extracellular scaffolds by simultaneously interacting with multiple postsynaptic ligands via LNS1-6 to enable synapse function (Miller et al., 2011; Chen et al., 2017). Despite this proposed role, a functional interrogation of extracellular sequences unique to $\alpha$-Nrxns has represented a daunting task due in large part to its length ( 1470 aa) and complexity (6 LNS domains, 3 EGF-like repeats and 6 alternative splice sites).

Given the length and complexity of $\alpha$-Nrxn extracellular sequences, our functional understanding of these molecules has almost entirely relied on $\alpha$-Nrxn constitutive knock-out (KO) mice. The constitutive ablation of all $\alpha$-Nrxns impaired presynaptic release at excitatory and inhibitory synapses and caused a reduction of somatic calcium currents (Missler et al., 2003; Zhang et al., 2005). Interestingly, the loss of individual $\alpha$-Nrxns resulted in distinct brain region and cell-type-specific phenotypes. Nrxn $1 \alpha$ KO mice exhibited impaired postsynaptic excitatory synaptic strength in hippocampus (Etherton et al., 2009). By contrast, Nrxn $3 \alpha$ KO mice did not have impaired excitatory synaptic strength in hippocampus but instead exhibited a significant reduction in presynaptic release probability at inhibitory synapses in olfactory bulb (Aoto et al., 2015). Together, these findings support the notion that individual $\alpha$-Nrxns participate in essential overlapping and non-overlapping functions at the synapse. Notably, although informative, the reported phenotypes are a result of the complete loss of all transsynaptic interactions (e.g., the extracellular scaffold) and intracellular signaling. While these studies demonstrated the functional importance of $\alpha$ Nrxns, the identification of the precise sequences necessary to enable these synaptic properties remains unknown.

One largely unpursued avenue to characterize the role of $\alpha$-Nrxn extracellular sequences is the interrogation of naturally occurring missense mutations. Mutations in exons that encode for $\alpha$-neurexin-specific extracellular sequences are linked to neu- 
ropsychiatric and neurodevelopmental disorders, strongly suggesting that these poorly understood $\alpha$-Nrxn extracellular sequences play a significant role in sculpting synapse function (Südhof, 2008; Reissner et al., 2013). Moreover, these extracellular mutations might serve as valuable tools to identify and address outstanding questions regarding how $\alpha$-neurexin extracellular sequences govern synapse function to ultimately provide novel functional insight that might otherwise be obscured in $\mathrm{KO}$ models. To this end, we identified a $\operatorname{Nrxn} 3 \alpha$ compound heterozygous patient diagnosed with profound intellectual disability and epilepsy and systematically interrogated a missense mutation that revealed a novel role for neurexin- $3 \alpha$ in controlling presynaptic morphology and neurotransmitter release at excitatory synapses.

\section{Materials and Methods}

\section{Experimental model and subject details}

Mouse generation and husbandry. All mouse work was approved by University of Colorado Denver Anschutz Medical Campus animal use committees. For all experiments, wild-type (WT) male and female mice (culture: P0, in vivo stereotactic injections: 3 weeks old, ex vivo slice: 5-6 weeks old) were used. Mice were kept on a $12 \mathrm{~h}$ light/dark cycle.

Patient genomic sequences were provided by GeneDx and use of the sequences was approved by University of Colorado School of Medicine (COMIRB: 16-0152) to Dr. Scott Demarest.

Analysis of culture hippocampal neurons. Hippocampi from P0 C57BL/6J were cultured on Matrigel-coated glass coverslips (Carolina Biological, 633009) as previously described (Aoto et al., 2013), infected with lentiviruses at $4 \mathrm{~d}$ in vitro (DIV) and analyzed at 13-15 DIV.

Virus preparations. AAVs and lentiviruses were engineered using the human synapsin promoter to drive expression. shRNA resistant mouse cDNAs were used for rescue constructs expressed from separate lentiviruses. Lentiviruses were made in HEK293T cells as previously described (Aoto et al., 2013). Adeno-associated viruses (AAVs) were prepared as previously described (Aoto et al., 2013).

Plasmids. The A687T mutation was introduced into mouse $\operatorname{Nrxn} 3 \alpha$ SS4+ and SS4 - cDNAs (XM_006515560.2) previously described (Aoto et al., 2015) by Gibson Assembly: F/R: ACCTGCGAAAGAGAGACATC TATCCTGAGCT/AGCTCAGGATAGATGTCTCTCTTTCGCA gGT. Gibson Assembly was used to insert mutant Nrxn3 $\alpha$ into FSW: F/R: TCGTGCCTGAGAGCGCAGTCTAGAGAATTCGCCACCATGAGCT TTAC/GATAAGCTTGAT ATCGAATTGTTAACGGATCCTTACACA TAATACTCCT and into AAV hSYN: F/R TGAGAGCGCAGTCGAG AAGGTACCGGATCCGCCACCATGAGCTTTACCCTCCACTCA/T TGATTATCGATAAGCTTGATATCGAATTCTTACACATAATACTC CTTGTCCTTG.

HEK aggregation assays were performed with cDNAs in pcDNA3.1 (Invitrogen) or pCMV. Gibson assembly was used to insert $\operatorname{Nrxn} 3 \alpha$ and NL1 and 3 into pcDNA3.1. Nrxn3 $\alpha$ F/R: TTTAAACTTAAGCTTGG TACCGAGCTCGGATCCGCCACCATGAGCTTTACCCTCCACTC/ GAGCGGCCGCCACTGTGCTGGATATCTGCAGAATTCTTACACA TAATACTCCTTGTCCTT; NL1 F/R: TAAACTTAAGCTTGGTACC GAGCTCGGCCACCATGGCACTTCCCAGATGCA TGTGG/CGCCA CTGTGCTGGATATCTGCAGAATTCTATACCCTGGTTGTTGAATG TGAA; NL3 was inserted into pcDNA3.1 at HindIII/XbaI using F/R primers: AAGCTTGCCACCATGTG GCTGCAGCCCTCGCTGT/TC TAGACTATACACGGGTAGTGGAGTGTGAGT.

Immunocytochemistry. Live surface immunolabeling of HA-tagged Nrxn $3 \alpha$ WT and A687T was performed as previously described (Aoto et al., 2013). Briefly, live neurons were washed with prewarmed PBS $0.5 \mathrm{~mm}$ $\mathrm{CaCl}_{2} 1 \mathrm{~mm} \mathrm{MgCl}_{2}$ and stained with mouse anti-HA (1:500; BioLegend) diluted in blocking buffer ( $2 \%$ normal goat serum; (Sigma-Aldrich) in PBS) for $5 \mathrm{~min}$ at $37^{\circ} \mathrm{C}$. Cells were washed three times with PBS for $5 \mathrm{~min}$ on ice before fixing with $4 \%$ PFA $+4 \%$ sucrose. Donkey anti-mouse Cy3-conjugated secondary antibody (1:200; Jackson ImmunoResearch; RRID:AB_2315777), diluted in blocking buffer, was applied for $45 \mathrm{~min}$ at room temperature.

Fixed staining of neurons was performed as previously described (Aoto et al., 2013). Briefly, neurons were fixed with $4 \%$ PFA and $4 \%$ sucrose, washed $3 \times$ with PBS before permeabilization with PBS $+0.2 \%$ Triton X-100. Neurons were then incubated for $45 \mathrm{~min}$ in blocking buffer $(2 \%$ normal goat serum $+0.2 \%$ Triton X-100 in PBS). Primary antibodies were applied to detect presynaptic proteins vGluT1 (1:1000; Synaptic Systems; RRID:AB_887878) and vGAT (1:500; Synaptic Systems; RRID:AB_887869), or postsynaptic markers: PSD95 (1:200; ThermoFisher; RRID:AB_325399) and Gephyrin (1:500; Synaptic Systems; RRID:AB_2619834). Secondary Cy2, Cy3 and Cy5-conjugated secondary antibodies (1:200; Jackson ImmunoResearch; RRID:AB_2307443; RRID:AB_2340813; RRID:AB_2492288) were applied then mounted on glass slides with Fluoromount-G (Southern Biotech) followed by imaging using spinning disk confocal microscopy at room temperature using a Zeiss Axio Observer microscope with a $63 \times$ oil-immersion objective Plan-Apo/1.4 NA objective, using 488, 594, and 647 laser excitation and a CSU-XI spinning-disk confocal scan head (Yokogawa) coupled to an Evolve 512 EM-CCD camera (Photometrics) controlled by SlideBook software. At least three coverslips per condition were imaged per session, and $\sim 15-25$ cells were acquired per condition. The same settings for laser power and photomultiplier were used to account for negligible bleed-through. During analysis, images were thresholded by intensity to exclude background noise and puncta were counted in the range of $0.25 \mu \mathrm{m}^{2}$.

qRT-PCR of Nrxn3 $\alpha$ mRNA in primary hippocampal cultures. Quantitative RT-PCR with $\beta$-actin as an internal control was used for mRNA measurements. mRNA was isolated using Quick-RNA Micro-Prep mRNA isolation kit (Zymo Research). qScript XLT 1-Step RT-qPCR ToughMix Kit (Quantabio) was used for RT. CFX384 Real-Time System RT-PCR instrument (Bio-Rad) was used to run and analyze reaction. TempPlate 384-Well Full-Skirt PCR, Single Notch polypropylene plates were used (USA Scientific). $\beta$-actin: F: GACTCATCGTACTCCTG CTTG, R: GATTACTGCTCTGGCT CCTAG, Probe: CTGGCCTCA CTGTCCACCTTCC. Nrxn $3 \alpha$ : F: TCCAGTTCAAGACCA CTTCAG, R: GTCACTGTTGCCTTTG ATCAC, Probe: TGgGACCATTGCCGA GATCA AACA. Nrxn3 $\beta$ : F: ACCACTCTGTGCCTATTTCTATC, R: TGTGCTGGGTCTGTCATTT G, Probe: TCGCTCCCCTGTTTCC CTTCG.

Culture electrophysiology. Whole-cell voltage-clamp recordings were made from pyramidal neurons, identified by the presence of dendritic spines (GFP fill), in all conditions. Recording pipettes were pulled to 3-4 $\mathrm{m} \Omega$ and series resistance was constantly monitored. Cells that experienced series resistance changes $>20 \%$ were discarded. Cultures were continuously superfused with room temperature ACSF (in mM): 126 $\mathrm{NaCl}, 2.5 \mathrm{KCl}, 1 \mathrm{NaH}_{2} \mathrm{PO}_{4}, 26.2 \mathrm{NaHCO}_{3}, 2.5 \mathrm{CaCl}_{2}, 1.3 \mathrm{MgSO}_{4} \bullet 7 \mathrm{H}_{2} \mathrm{O}$, $11 \mathrm{D}$-glucose, $\sim 290 \mathrm{mOsm}$. The internal solution consisted of the following (in mM): 115 Cs-methanesulfonate, $15 \mathrm{CsCl}, 8 \mathrm{NaCl}, 0.2 \mathrm{EGTA}, 10$ HEPES, 4 Mg-ATP, $0.3 \mathrm{Na}$-GTP, 10 TEA-Cl, $10 \mathrm{Na}_{2}$-phosphocreatine, 1 $\mathrm{MgCl}_{2}, \sim 290 \mathrm{mOsm}$. Cells were held at $-70 \mathrm{mV}$ and mIPSCs $(0.5 \mu \mathrm{M}$ tetrodotoxin, $0.5 \mu \mathrm{M}$ NBQX, and $50 \mu \mathrm{M}$ D-AP5) and mEPSCs $(0.5 \mu \mathrm{M}$ tetrodotoxin and $100 \mu \mathrm{M}$ picrotoxin) were recorded. Miniature events were handpicked and analyzed in Clampfit 10 (Molecular Devices) using template matching and a threshold of $5 \mathrm{pA}$. Evoked synaptic currents were elicited with a homemade nichrome bipolar stimulating electrode placed $100-150 \mu \mathrm{m}$ from the soma of recorded neurons, using a Model 2100 Isolated Pulse Stimulator (A-M Systems) controlled by Clampex 10 data acquisition software (Molecular Devices). After formation of the whole-cell configuration and equilibration of the internal solution, the series resistance was compensated to 5-8 M $\Omega$. For AMPAR responses, cells were held at $-70 \mathrm{mV}$ and recorded in the presence of picrotoxin $(100 \mu \mathrm{M})$ D-AP5 $(50 \mu \mathrm{M})$. For evoked NMDAR, cells were held at +40 $\mathrm{mV}$ and recorded in NBQX $(5 \mu \mathrm{M})$. Evoked IPSCs were measured at -70 $\mathrm{mV}$ in the presence of D-APV $(50 \mu \mathrm{M})$ and NBQX (5 $\mu \mathrm{M})$. Presynaptic release probability was measured during paired pulse recordings at interstimulus intervals (ISIs) ranging from 20 to $1000 \mathrm{~ms}$. Cells that did not exhibit short-term plasticity at all short ISIs $(20-100)$ were discarded from further analysis. For all evoked measurements, QX-314 (5 mM) was added to the internal to prevent firing. For all experiments, the experimenter was blind to the recording condition.

Stereotactic injections. Stereotactic injections were performed as previously described (Liu et al., 2019). Briefly, P21 WT C57BL/6J mice were 
deeply anesthetized with tribromoethanol (200 mg/kg). Using a syringe pump (WPI), $1 \mu \mathrm{l} \mathrm{AAV}$ was injected bilaterally into CA1 at a rate of 14 $\mathrm{ml} / \mathrm{h}$. The pulled glass micropipette (WPI) was held in place for $5 \mathrm{~min}$ to prevent backflow before being slowly withdrawn. Coordinates for CA1 (from bregma): AP: -3.2 , ML: \pm 3.45 , DV: -2.5 (relative to pia). Efficiency and localization of infection was assessed mRuby fluorescence in CA1 on day P35. Stitched images were acquired using an Olympus VS120 slide-scanning microscope with Olympus VS-ASW software.

\section{Acute slice electrophysiology}

Whole-cell recording in subiculum was performed on $300 \mu \mathrm{m}$ thick acute slices from WT mice coinfected with AAV encoding shRNA and mRuby or shRNA + Nrxn3 $\alpha^{\text {WT/A687T }}$ SS4 - into CA1 were performed in vivo by stereotactic injection on $\mathrm{P} 21$ mice.

Slices were made in cold cutting solution containing the following (in mM): $85 \mathrm{NaCl}, 75$ sucrose, $2.5 \mathrm{KCl}, 1.3 \mathrm{NaH}_{2} \mathrm{PO}_{4}, 24 \mathrm{NaHCO}_{3}, 0.5 \mathrm{CaCl}_{2}$, $4 \mathrm{MgCl} \bullet 6 \mathrm{H}_{2} \mathrm{O}$, and $25 \mathrm{D}$-glucose. Slices were allowed to recover at $31.5^{\circ} \mathrm{C}$ for $30 \mathrm{~min}$ then at room temperature for $1 \mathrm{~h}$ in oxygenated ACSF (same as culture electrophysiology). Acute slices where transferred to a recording chamber continuously superfused with oxygenated ACSF $(1.5 \mathrm{ml} /$ min) maintained at $29.5^{\circ} \mathrm{C}$. A potassium gluconate internal was used to allow for the depolarization and spiking identification of regular versus burst spiking cells in subiculum (in mM): $140 \mathrm{~K}$-gluconate, $5 \mathrm{KCl}, 10$ HEPES, 4 ATP- $\mathrm{Mg}^{2+}, 0.5$ GTP-Na ${ }^{+}, 10$ phosphocreatine, 0.2 EGTA. Series resistance was constantly monitored and cells that experienced changes of $>20 \%$ were discarded. Cells that did not exhibit an AMPARmediated EPSC I/O relationship were excluded. AMPAR-mediated EPSCs were recorded at $-70 \mathrm{mV}$ and evoked using a homemade nichrome electrode controlled by an A-M Systems Isolated Pulse Stimulator in the presence of D-AP5 (50 $\mu \mathrm{M})$ and PTX (100 $\mu \mathrm{M})$. Paired-pulse ratios (PPRs) were measured with ISIs of $40 \mathrm{~ms}$. LTP was induced in regular and burst spiking cells in current-clamp mode by stimulating 4 times at $100 \mathrm{~Hz}$ with $10 \mathrm{~s}$ intertrain intervals and recorded in voltageclamp in the presence of PTX $(100 \mu \mathrm{M})$. EPSCs during baseline and post-LTP induction were sampled at $0.1 \mathrm{~Hz}$ and binned per minute. The magnitude of LTP was determined by comparing the average EPSC amplitude of 50-60 min post-induction to the average amplitude of $10^{\prime}$ baseline in each experiment. PPRs were assessed before LTP induction and 60 min post-induction.

All synaptic currents were monitored with a MultiClamp 700B amplifier and digitized with a Digidata 1440A or 1550B (Molecular Devices). Data were collected at $10 \mathrm{kHz}$ and filtered with a low-pass filter at $2 \mathrm{kHz}$. For all experiments, the experimenter was blind to the recording condition.

\section{Scanning electron microscopy}

Cells were fixed on the coverslips in $2.5 \%$ glutaraldehyde in $0.1 \mathrm{~m}$ sodium cacodylate buffer. Cells were first rinsed three times in $0.1 \mathrm{M}$ sodium cacodylate buffer, $\mathrm{pH} 7.4$, then postfixed in $1 \%$ osmium tetroxide and $1.5 \%$ potassium ferrocyanide for $30 \mathrm{~min}$. After three washes in water, the cells were immersed in $1 \%$ uranyl acetate in water for $30 \mathrm{~min}$. Following two rinses in water, they were dehydrated through a graded ethanol series $(50,70,95$, and $100 \% \times 2)$ for 5 min each. The coverslips were quickly blotted and a drop of Epon/Araldite resin was applied to the coverslips before prefilled BEEM capsules were flipped over on top of them. Samples were polymerized for $24 \mathrm{~h}$ at $60^{\circ} \mathrm{C}$ in an oven. The coverslips were then removed by dipping the block in liquid nitrogen. Ultrathin sections $(60 \mathrm{~nm})$ were cut on a Reichert Ultracut $S$ from a small trapezoid positioned over the cells and were picked up on Formvar-coated slot grids (EMS). Sections were imaged on a FEI Tecnai G2 transmission electron microscope with an AMT digital camera. Vesicles were counted on electron micrographs using Reconstruct software. A vesicle was considered "docked" if it made direct contact with the presynaptic membrane as described by Bacaj et al. (2015). Vesicles within $100 \mathrm{~nm}$ distance from the presynaptic membrane were considered to be in the "putative active zone". Length of active zone (AZ) was measured as the length of the presynaptic membrane opposite to the putative postsynaptic density (PSD). The length of the putative PSD was determined by enhancing contrast on Reconstruct to identify boundary of PSD seen as increased cytoplasmic opacity and thickness bordering the postsynaptic membrane. Three independent cultures were analyzed by a blinded experimenter.

\section{HEK cell aggregation study}

HEK293T cells expressing either GFP alone or together with LRRTM2 or neuroligin- 1 were mixed in a 1:1 ratio with cells expressing mCherry alone or together with neurexin-3 $\alpha^{\text {WT/A687T }}$ with or without a splice insert in SS4, and incubated at room temperature for 30-60 $\mathrm{min}$ in DMEM-containing the following (in $\mathrm{mm}$ ): 50 HEPES- $\mathrm{NaOH}, \mathrm{pH}$ 7.4, $10 \% \mathrm{FBS}, 10 \mathrm{MgCl}_{2}$, and $10 \mathrm{CaCl}_{2}$. Cells were imaged by fluorescence microscopy using a wide-field Zeiss Axio Vert 200M with an Olympus $5 \times$ air objective (NA: 0.55; WD: $26 \mathrm{~mm}$ ). Extent of aggregation was quantified using MATLAB scripts that calculated the percentage overlap of green (GFP) on red (mCherry) puncta.

\section{Quantification and statistical analyses}

Statistical analysis was performed using GraphPad Prism 7 and Microsoft Excel. Distribution of data was evaluated for normality using ShapiroWilkes test. Additional analyses included independent unpaired $t$ tests, and ANOVAs. Correction for multiple comparisons was also used where appropriate. Kolmogorov-Smirnov test was used to analyze the statistical significance of cumulative probabilities. Outliers were identified based on a predefined criteria using the ROUT method of Prism, where $Q=1 \%$. Outlier analysis was performed on immunocytochemistry experiments to identify synaptic clusters comprised of multiple overlapping puncta, which if included in the analysis would artificially skew the data toward larger puncta sizes. All bar graphs represent the mean from at least three independent experiments except where noted. Data values reported are given as mean \pm SEM. All bar graphs represent the mean from at least three independent experiments except where noted.

\section{Contact for reagent and resource sharing}

Further information and requests for resources and reagents should be directed to and will be fulfilled by the corresponding author.

\section{Results}

\section{The A687T mutation does not impair surface trafficking of $\operatorname{Nrxn} 3 \alpha$}

Whole exome sequencing revealed a truncating mutation in the paternal allele (p1076dupC, NM_001272020.1) which is similar to a nonfunctional secreted isoform previously tested (Aoto et al., 2015), and a missense mutation in the maternal allele (pA310T, NM_004796.5) in a sequence located between EGF2 and LNS4 of $\operatorname{Nrxn} 3 \alpha$ that is conserved between $\alpha$ neurexins and throughout vertebrate evolution (Fig. $1 A$ ). In mice, the orthologous mutation is $\mathrm{A} 687 \mathrm{~T}\left(\mathrm{Nrxn} 3 \alpha^{\mathrm{A} 687 \mathrm{~T}}\right)$. Intriguingly, mutations in this linker region of other neurexins, including a rare missense mutation in Nrxn $1 \alpha$ linked to autism spectrum disorder located immediately upstream of the residue studied here (Fig. 1A, highlighted in blue; Yan et al., 2008), suggests that this unstudied sequence of residues is important for the synaptic function of neurexins.

We first asked whether the A687T mutation alters the surface availability of $\operatorname{Nrxn} 3 \alpha$. Although unlikely due to the location of the mutation in a linker between two structured domains, the alanine-to-threonine mutation might adversely affect trafficking of $\mathrm{Nrxn} 3 \alpha^{\text {A687T }}$, which would effectively recapitulate a $\mathrm{KO}$ allele. Reliable antibodies that detect $\operatorname{Nrxn} 3 \alpha$ do not exist, therefore, we designed lentiviruses that encoded for extracellular HA-tagged $\operatorname{Nrxn} 3 \alpha$ to quantify surface $\operatorname{Nrxn} 3 \alpha$ levels (Fig. $1 B$ ). We positioned the extracellular HA tag in the $5^{\prime}$ end of exon 24, the last coding exon for Nrxn3, using a strategy previously reported for Nrxn1 (Pak et al., 2015 their p. 1). The introduction of the HA tag in this locus does not alter surface trafficking of endogenous protein (Pak et al., 2015). We transduced primary hippocampal neurons at $4 \mathrm{DIV}$ with lentivirus expressing HA-tagged $\operatorname{Nrxn} 3 \alpha^{\mathrm{WT}}$ or 
$\operatorname{Nrxn} 3 \alpha^{\text {A687T }}$ with or without the splice-site 4 exon (SS4+ or SS4-), and then performed live anti-HA surface labeling at 14 DIV. We manipulated SS4 because the presence or absence of the alternative splice-site 4 exon regulates binding to almost all known neurexin ligands and is differentially expressed in excitatory and inhibitory synapses (Aoto et al., 2015; Fuccillo et al., 2015; Nguyen et al., 2016). We found that the mutation did not alter surface expression relative to WT for either splice-site 4 isoform (integrated fluorescence intensities, WT SS4-: $8062 \pm$ $1353.7 n=18$; A687T SS4-: $8846 \pm 1986.1 n=14, p=0.946$; WT SS4+: $8779 \pm 1239.7 n=18$; A687T SS4+: $9466 \pm 1571.2$ $n=18, p=0.733$; one-way ANOVA multiple comparisons; Fig. $1 C)$. Importantly, we observed little surface HA immunoreactivity for WT and mutant Nrxn $3 \alpha$ in dendrites, but observed significant immunofluorescence in axons and in axon varicosities opposed to postsynaptic spines, indicating that the mutation did not impair axon trafficking (Fig. $1 C$, arrowheads). Importantly, transduction of neurons with neurexin- $3 \alpha$ expressing lentivirus rescued synaptic function in Nrxn3 mutant mice (Aoto et al., 2013), which strongly suggests that a sufficient fraction of exogenous $\operatorname{Nrxn} 3 \alpha$ localizes to presynaptic AZs to govern function. We next asked whether this mutation impacts synapse morphology and/or function.

\section{Generation and functional characterization of the molecular replacement approach}

To mitigate potential confounds caused by overexpression artifacts and by the presence of endogenous $\operatorname{Nrxn} 3 \alpha$, we used a molecular replacement approach that consisted of lentivirusmediated shRNA knockdown of endogenous $\operatorname{Nrxn} 3 \alpha$ and replacement with exogenous WT or A687T Nrxn3 $\alpha$ (Fig. 2A). Lentivirus-mediated transduction is highly efficient in primary neuron cultures and routinely results in the infection of $>95 \%$ of all neurons, which is essential when studying the functional role of presynaptic molecules (Aoto et al., 2013). We tested three Nrxn $3 \alpha$ shRNAs that targeted distinct regions of mRNA unique to Nrxn $3 \alpha$ and found that each shRNA knocked down endogenous Nrxn $3 \alpha$ mRNA by $70-80 \%$ (left; Nrxn3 $\alpha$ relative to GFP control: shRNA1: $0.88 \pm 0.055, n=3, p=0.0001$; shRNA2: $0.73 \pm 0.096, n=3, p=0.0002$; shRNA3: $0.66 \pm 0.080, n=3$, $p=0.0004$; one-way ANOVA multiple comparisons; Fig. $2 B)$. Of the three shRNAs tested, we chose to use shRNA1 because it displayed the greatest knockdown of endogenous $\mathrm{Nrxn} 3 \alpha$ mRNA. Replacement with shRNA-resistant $\operatorname{Nrxn} 3 \alpha^{\text {WT }}$ or Nrxn3 $\alpha^{\text {A687T }}$ SS4 + or SS4 - resulted in a $\sim$ fourfold increase in mRNA levels (shRNA: $0.2912 \pm 0.04317 n=4$; shRNA/WT SS4 $+4.643 \pm 0.2135 n=4$; shRNA/A687T SS4 + 3.265 \pm 0.8536 , $n=4 ;$ shRNA/WT SS4 $-3.668 \pm 0.8963, n=4 ;$ shRNA/A687T SS4 $-3.337 \pm 1.066, n=4$; shRNA vs shRNA/WT SS4-: $p=$ 0.0035 ; shRNA vs shRNA/A687T SS4 $-: p=0.0084$; shRNA vs shRNA/WT SS4+: $p=0.0003$; shRNA vs shRNA/A687T SS4+: $p=0.0102$; one-way ANOVA multiple comparisons; Fig. 2B). Importantly, none of the Nrxn $3 \alpha$ shRNAs tested altered mRNA levels of Nrxn $3 \beta$ (GFP control: $1 \pm 0$ fold-change, $n=4$; shRNA: $1.179 \pm 0.1585$ fold-change, $n=4$; shRNA/WT SS4 $+: 1.183 \pm$ 0.2973 fold-change, $n=4$; shRNA/A687T SS4 $+: 1.15 \pm 0.1863$ fold-change, $n=4$; shRNA/WT SS4-: $1.163 \pm 0.2299$ foldchange, $n=4$; shRNA/A687T SS4-: $1.276 \pm 0.1551$ fold-change, $n=4, p=0.9522$; one-way ANOVA multiple comparisons; Fig. $2 B$, right).

In contrast to the $\mathrm{Nrxn} 3 \alpha / \beta \mathrm{cKO}$, it has been previously demonstrated in primary hippocampal cultures that constitutive ablation of $\operatorname{Nrxn} 3 \alpha$ does not alter excitatory or inhibitory synapse function (Aoto et al., 2015). To test for off-target functional effects produced by shRNA, we virally transduced primary hippocampal neurons with shRNA1 or control lentivirus and systematically measured inhibitory and excitatory synaptic transmission parameters using whole-cell voltage-clamp electrophysiology. Membrane capacitance $\left(C_{\mathrm{m}}\right)$ and membrane resistance $\left(R_{\mathrm{m}}\right)$, monitored during IPSC recordings were unchanged following knockdown of $\operatorname{Nrxn} 3 \alpha\left(C_{\mathrm{m}}\right.$ : GFP control: $115.4 \pm 6.869$ pF, $n=29$; shRNA: $130.6 \pm 8.547 \mathrm{pF}, n=28 ; p=0.1661 ; R_{\mathrm{m}}$ : GFP control: $235 \pm 18.32 \mathrm{M} \Omega, n=29$; shRNA: $258.1 \pm 19.31$ $\mathrm{M} \Omega, n=28 ; p=0.3926$; Student's $t$ test; Fig. $2 C$ ) or EPSC recordings $\left(C_{\mathrm{m}}\right.$ : GFP control: $157.9 \pm 5.674 \mathrm{pF}, n=31$; shRNA: $144.8 \pm 6.21 \mathrm{pF}, n=31 ; p=0.1283 ; R_{\mathrm{m}}$ : GFP control: $160.3 \pm$ 7.422 M $\Omega, n=31$; shRNA: $171.1 \pm 8.869 \mathrm{M} \Omega, n=31 ; p=$ 0.3518 ; Student's $t$ test; Fig. $2 G$ ). Additionally, the frequency and amplitude of miniature IPSCs [(mIPSCs) frequency, GFP control: $1.593 \pm 0.1998 \mathrm{~Hz}, n=29$; $\operatorname{shRNA}: 1.181 \pm 0.1783 \mathrm{~Hz} ; p=$ 0.1307; amplitude, GFP control: $14.94 \pm 0.6115 \mathrm{pA}, n=29$; shRNA: $14.16 \pm 0.6757 \mathrm{pA}, n=28 ; p=0.3929]$ and miniature EPSCs [(mEPSCs) frequency, GFP control: $0.793 \pm 0.1948 \mathrm{~Hz}$, $n=41$; shRNA: $0.6178 \pm 0.21 \mathrm{~Hz}, n=43 ; p=0.5736$; amplitude, GFP control: $15.39 \pm 1.136 \mathrm{pA}, n=41$; shRNA $14.26 \pm 0.9563$ $\mathrm{pA}, n=43$; $p=0.4751$; Student's $t$ test) of shRNA1 infected neurons were similar to the mock infected control condition (Fig. $2 D, H$ ), which is consistent with the $\operatorname{Nrxn} 3 \alpha \mathrm{KO}$ mouse (Aoto et al., 2015).

Recent studies suggest that spontaneous and action potential evoked release may represent mechanistically distinct processes (Ramirez and Kavalali, 2011; Crawford et al., 2017; Chanaday and Kavalali, 2018). Thus, we tested whether the acute knockdown of Nrxn3 $\alpha$ alters action-potential evoked inhibitory or excitatory synaptic transmission. We observed no overt differences in electrically evoked IPSC amplitudes (GFP control: $1.774 \pm$ $0.07477 \mathrm{nA}, n=30$; shRNA: $1.299 \pm 0.2054 \mathrm{nA}, n=24 ; p=$ 0.0955; Student's $t$ test) or IPSC PPRs ( $40 \mathrm{~ms}$ ISI: GFP control: $0.5507 \pm 0.02025, n=30$; shRNA: $0.6012 \pm 0.04813, n=24 ; p=$ 0.3886 ; Student's $t$ test) between control and shRNA1 conditions (Fig. 2E,F). The only PPR ISI that exhibited a significant difference was at $50 \mathrm{~ms}$ (GFP control: $0.5505 \pm 0.02213, n=30$; shRNA: $0.6314 \pm 0.0126, n=24 ; p=0.0337$ Student's $t$ test), however, we failed to detect a similar change measuring PPRs at shorter ISIs. Additionally, reduced IPSC PPR at a $50 \mathrm{~ms}$ ISI, indicative of increased release probability, is incongruent with the reported mIPSC frequency and the slightly reduced evoked IPSC amplitudes observed for shRNA1. Similarly, AMPAR-mediated (GFP control: $0.7181 \pm 0.05714 \mathrm{nA}, n=50$; shRNA: $0.6066 \pm$ $0.06402 \mathrm{nA}, n=32 ; p=0.2635$; Student's $t$ test) and NMDARmediated evoked EPSC amplitudes (GFP control: $0.5026 \pm$ $0.06404 \mathrm{nA}, n=17$; shRNA: $0.5081 \pm 0.09263 \mathrm{nA}, n=15 ; p=$ 0.9602; Student's $t$ test) and NMDAR PPRs (40 ms ISI: GFP control: $0.572 \pm 0.05819, n=17$; shRNA: $0.6344 \pm 0.0931, n=$ $15 ; p=0.5691$; Student's $t$ test) were unaltered by shRNA1 (Fig. $2 I-K)$. Together, these data suggest that knockdown of $\operatorname{Nrxn} 3 \alpha$ by shRNA1 does not alter spontaneous or action potentialevoked inhibitory or excitatory neurotransmission. Thus, for all molecular replacement experiments in vitro and in vivo, we used shRNA1 alone as an infection control.

\section{Molecular replacement with Nrxn $3 \alpha^{\mathrm{A687T}}$ SS4- selectively increases presynaptic vGluT1 cluster size in vitro}

To examine whether the A687T mutation alters synaptic morphology, we fixed and stained 14 DIV primary hippocampal cul- 
A

Molecular Replacement Strategy

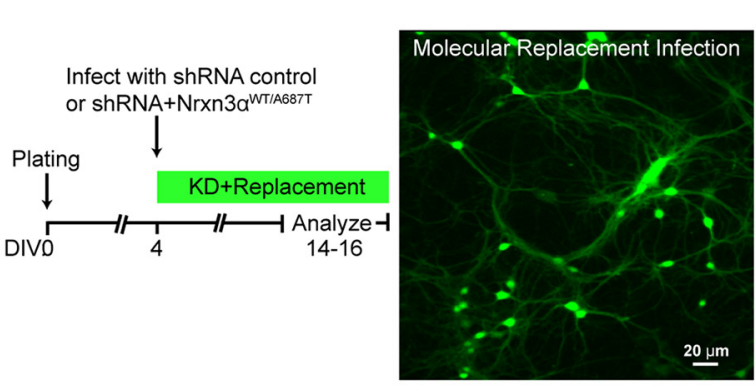

B

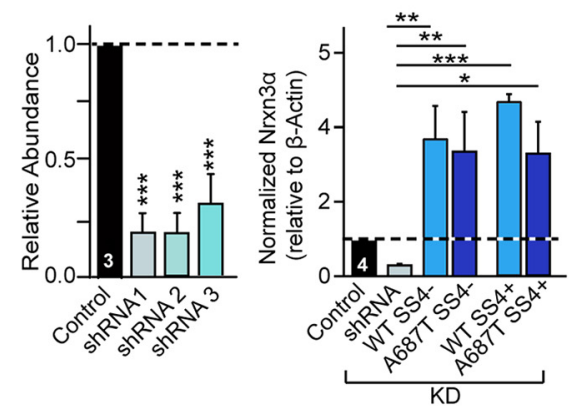

Nrxn3 $\alpha$ shRNA - mRNA Levels

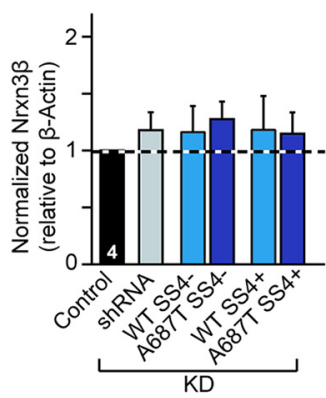

Inhibitory synaptic transmission - Nrxn3a Knockdown vs. Control

C $_{\text {Passive Membrane Properties }}$

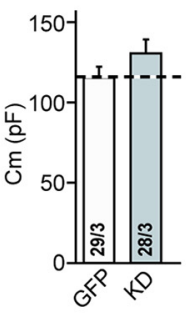

E Evoked IPSCs

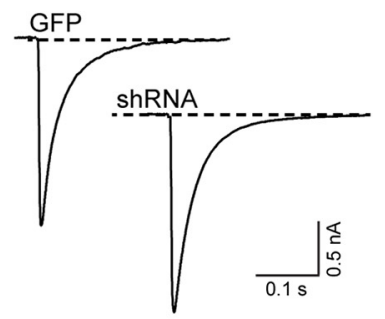

D

Miniature Inhibitory Postsynaptic Currents

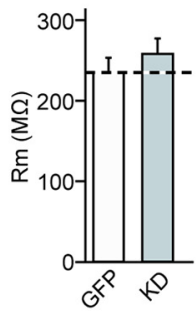

Control (GFP)

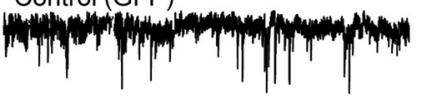

$K D$

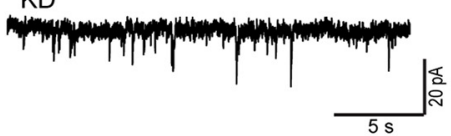

$\mathbf{F}$
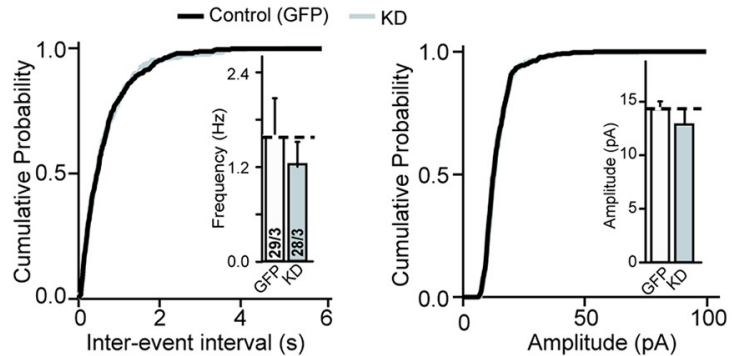

IPSC Paired-pulse Ratios
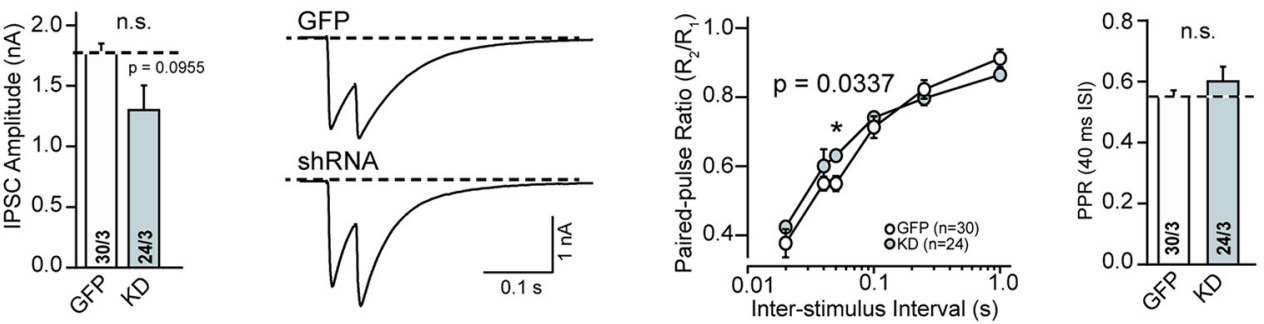

Excitatory synaptic transmission - Nrxn3a Knockdown vs. Control

G Passive Membrane Properties $\mathbf{H}$
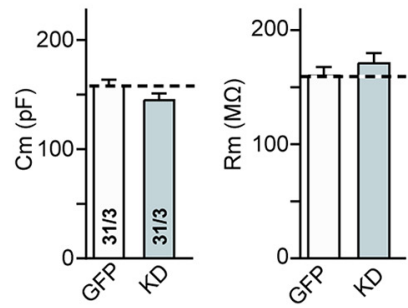

I Evoked AMPAR EPSCs<smiles></smiles>

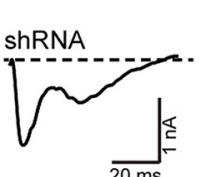

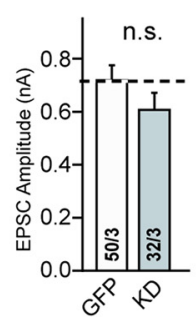

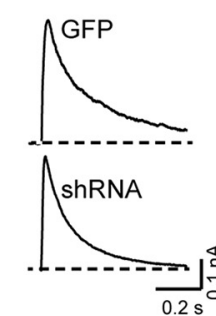

Control (GFP)

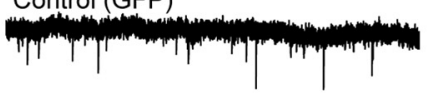

$\mathrm{KD}$

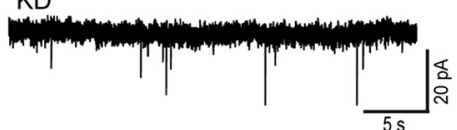

K

\section{Evoked NMDAR EPSCs}
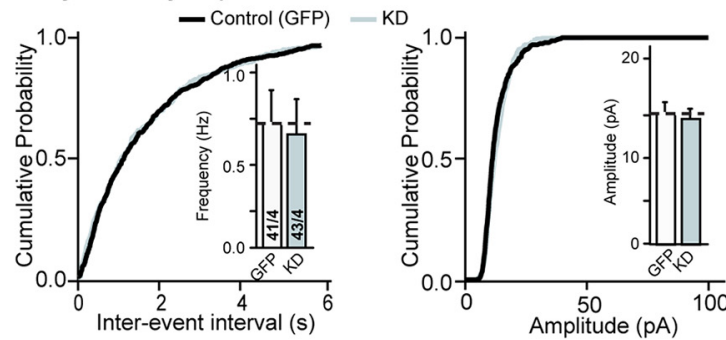

NMDAR Paired-pulse Ratios
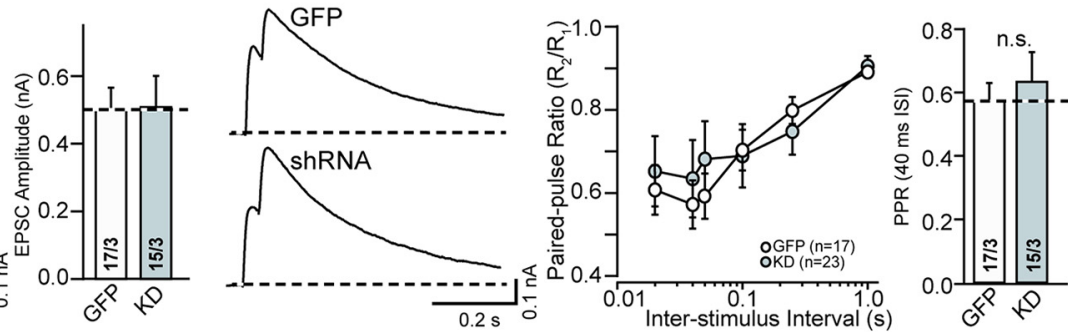

Figure 2. Knockdown of neurexin-3 $\alpha$ does not alter inhibitory or excitatory synaptic transmission. $\boldsymbol{A}$, Schematic illustration of the molecular replacement approach (left) and representative image of lentivirus transduced hippocampal culture neurons (right). Note: nearly all neurons are infected with lentivirus, which is critical when studying the function of presynaptic proteins. $\boldsymbol{B}$, qRT-PCR after lentivirus knockdown of Nrxn3 $\alpha$ with three different shRNAs (left), mRNA levels after molecular replacement with SS4 is oforms of Nrxn3 $\alpha$ wT or Nrxn3 $\alpha{ }^{\text {A687T }}$ (middle), and Nrxn3 $\beta$

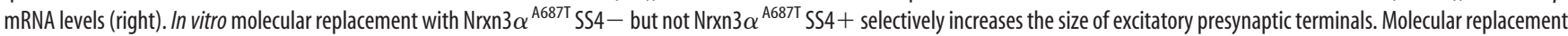
experiments were performed using lentivirus to introduce SS4 isoforms of WT and A687T Nrxn3 $\alpha$ as described in $\boldsymbol{A}$. C $\boldsymbol{F}$, Electrophysiological interrogation of inhibitory (Figure legend continues.) 
tures following lentivirus-mediated molecular replacement with both SS 4 isoforms of $\mathrm{Nrxn} 3 \alpha^{\text {WT }}$ or $\mathrm{Nrxn} 3 \alpha^{\mathrm{A} 687 \mathrm{~T}}$ at $4 \mathrm{DIV}$. To investigate excitatory and inhibitory postsynaptic morphology, we immunostained primary hippocampal neurons with antibodies to visualize gephyrin and PSD-95, which are postsynaptic markers for inhibitory and excitatory synapses, respectively (Fig. $3 A$ ). We found that the density, intensity, and size of the gephyrin and PSD95-immunoreactive clusters were unaltered by replacement with either SS4 isoform of $\operatorname{Nrxn} 3 \alpha^{\mathrm{A} 687 \mathrm{~T}}$ (gephyrin density, shRNA: $6.7 \pm 1.3, n=64 / 3$; shRNA/WT SS4-: $6.2 \pm 1.5, n=$ 63/3; shRNA/A687T SS4-: $6.2 \pm 1.4, n=67 / 3$; shRNA/WT SS4+: $6.2 \pm 1.0, n=67 / 3$; shRNA/ A687T SS4+: $5.6 \pm 0.5, n=$ 57/3; intensity, shRNA: $42.3 \pm 5.3 \mathrm{AU}$; shRNA/WT SS4-: $40.9 \pm$ 5.0 AU; shRNA/ A687T SS4-: $43.1 \pm 6.1$ AU; shRNA/WT SS4+: $44.2 \pm 6.5$; shRNA/A687T SS4 $+: 45.6 \pm 6.3$; size, shRNA: $0.78 \pm$ $0.06 \mu \mathrm{m}^{2}$; shRNA/WT SS4-: $0.70 \pm 0.06 \mu \mathrm{m}^{2}$; shRNA/A687T SS4-: $0.8 \pm 0.13 \mu \mathrm{m}^{2}$; shRNA/WT SS4+: $0.80 \pm 0.07 \mu \mathrm{m}^{2}$; shRNA/A687T SS4+: $0.78 \pm 0.12 \mu \mathrm{m}^{2} ; p=0.99$; one-way ANOVA, multiple comparisons; Fig. $3 B, C$ ). Next, we quantified presynaptic morphology using antibodies to detect the inhibitory marker vGAT and the excitatory marker vGluT1, following molecular replacement with $\operatorname{Nrxn} 3 \alpha^{\mathrm{WT}}$ or $\operatorname{Nrxn} 3 \alpha^{\mathrm{A} 687 \mathrm{~T}}$ (Fig. 3D). Neither SS4 isoform of Nrxn3 $\alpha^{\text {A687T }}$ altered vGAT cluster density, quantified as the number of clusters per unit length of dendrite, intensity or size (density, shRNA: $3.1 \pm 0.18, n=77 / 3$; shRNA/WT SS4-: $2.75 \pm 0.42, n=72 / 3$; shRNA/A687T SS4-: $3.7 \pm 0.02, n=81 / 3$; shRNA/WT SS4+: $2.8 \pm 0.19, n=61 / 3$; shRNA/A687T SS4+: $2.8 \pm 0.35, n=59 / 3$; intensity, shRNA: $43.3 \pm 9.1 \mathrm{AU}$; shRNA/WT SS4-: $44.6 \pm 9.8 \mathrm{AU}$; shRNA/A687T SS4-: $42.5 \pm 7.6 \mathrm{AU}$; shRNA/WT SS4+: $41.4 \pm 8.2 \mathrm{AU}$; shRNA/ A687T SS4+: $41.7 \pm 7.0$ AU; size, shRNA: $0.63 \pm 0.033 \mu \mathrm{m}^{2}$; shRNA/WT SS4-: $0.63 \pm 0.030 \mu \mathrm{m}^{2}$; shRNA/A687T SS4-: $0.72 \pm 0.16 \mu \mathrm{m}^{2}$; shRNA/WT SS $4+: 0.63 \pm 0.040 \mu \mathrm{m}^{2}$; shRNA/ A687T SS4+: $0.53 \pm 0.033 \mu \mathrm{m}^{2} ; p>0.05$; one-way ANOVA, multiple comparisons; Fig. $3 E$ ). However, we observed a striking $\sim 50 \%$ increase in the size of vGluT1 clusters after molecular replacement with $\operatorname{Nrxn} 3 \alpha^{\text {A687T }}$ SS4- without alterations in vGluT1 cluster density and intensity relative to controls (density, shRNA: $4.7 \pm 0.24, n=77 / 3$; shRNA/WT SS4 $-: 4.54 \pm 0.50, n=$

$\leftarrow$

(Figure legend continued.) synapse function in cultured hippocampal neurons following mock lentivirus infection or infection with Nrxn3 $\alpha$ shRNA lentivirus. $C, C_{\mathrm{m}}$ and $R_{\mathrm{m}}$ of neurons used for IPSC measurements were unchanged between conditions. $D$, mIPSC frequency and amplitudes were unchanged between mock infected to Nrxn3 $\alpha$ shRNA infected cultured hippocampal neurons. Representative mIPSC traces (left) and summary graphs (right) of mIPSC frequency and amplitudes at 13-15 DIV. E, Electrically evoked IPSCs were not altered by acute knockdown of Nrxn3 $\alpha$. Evoked IPSCs traces (left) and summary graph (right) of the evoked IPSC amplitudes at 13-15 DIV. $\boldsymbol{F}$, Inhibitory PPRs, an assessment of presynaptic release, are unchanged after acute knockdown of Nrxn3 $\alpha$. Representative traces (left) and summary graphs (middle, right) of IPSC PPR amplitude monitored in cultured hippocampal neurons at 13-15 DIV. G-K, Electrophysiological interrogation of excitatory synapse function in cultured hippocampal neurons following mock lentivirus infection or infection with Nrxn3 $\alpha$ shRNA lentivirus. $G, C_{\mathrm{m}}$ and $R_{\mathrm{m}}$ of neurons used for EPSC measurements were unchanged between conditions. $\boldsymbol{H}, \mathrm{mEPSC}$ frequency and amplitudes were unchanged between mock infected to $\mathrm{Nrxn} 3 \alpha$ shRNA infected cultured hippocampal neurons. Representative mEPSC traces (left) and summary graphs (right) of mEPSC frequency and amplitudes at 13-15 DIV. I-J, Electrically evoked EPSCs were not altered by acute knockdown of Nrxn3 $\alpha$. Evoked EPSCs traces (left) and summary graph (right) of the evoked AMPA-mediated EPSC amplitudes at 13-15 DIV (I) and NMDARmediated ( $\boldsymbol{(})$. $\boldsymbol{K}$, Same as $\boldsymbol{F}$ except NMDAR PPRs were assessed. Data shown are mean \pm SEM; mean values calculated from the average of the number of total experiments. Numbers in bars represent cells/number of experiments. Statistical significance was determined by single-factor ANOVA, multiple comparisons for $\boldsymbol{A}$ and $\boldsymbol{B}$ and Student's $\boldsymbol{t}$-test for $\boldsymbol{C}-\boldsymbol{K}$. n.S., not significant, ${ }^{*} p<0.05 ;{ }^{* *} p<0.01 ;{ }^{* * *} p<0.001$.
72/3; shRNA/A687T SS4-: $4.98 \pm 0.23, n-81 / 3$; shRNA/WT SS4+: $5.23 \pm 0.46, n=61 / 3$; shRNA/A687T SS4+: $4.48 \pm 0.20$, $n=59 / 3 ; p>0.05$; intensity, shRNA: $52.3 \pm 11.5 \mathrm{AU}$; shRNA/WT SS4-: $53.1 \pm 13.8 \mathrm{AU} ;$ shRNA/A687T SS4-: $49.0 \pm$ $11.8 \mathrm{AU}$; shRNA/WT SS4+: $52.0 \pm 13.9 \mathrm{AU} ;$ shRNA/A687T SS4+: $50.3 \pm 12.7$ AU; $p>0.05$; size, shRNA: $0.84 \pm 0.090 \mu \mathrm{m}^{2}$; shRNA/WT SS4-: $0.90 \pm 0.040 \mu \mathrm{m}^{2}$; shRNA/A687T SS4-: $1.29 \pm 0.066 \mu \mathrm{m}^{2}$; shRNA/WT SS4+: $0.81 \pm 0.072 \mu \mathrm{m}^{2}$; shRNA/A687T SS4+: $0.89 \pm 0.069 \mu \mathrm{m}^{2}$; shRNA vs shRNA/ A687T SS4-: $p=0.0085$; shRNA/WT SS4 - vs shRNA/A687T SS4- $p=0.02$; shRNA/WT SS4 + vs shRNA/A687T SS4 $-: p=$ 0.005; shRNA/A687T SS4+ vs shRNA/A687T SS4- $p=0.018$ one-way ANOVA, multiple comparisons; Fig. $3 F$ ). This intriguing increase in vGluT1 cluster size suggests that in excitatory boutons there are more vesicles of neurotransmitter and/or more vGluT1 molecules per vesicle. By contrast, the inclusion of the SS4 exon (SS4+) did not alter the density, intensity or size of vGluT1-immunoreactive clusters (Fig. $3 F$ ). Unexpectedly, these data suggest that the A687T mutation does not impact inhibitory synapse morphology but exhibits a selective splice-site 4-dependent increase in the abundance of vGluT1 in excitatory presynaptic terminals, a phenotype distinct from those previously observed in the Nrxn $3 \alpha / \beta$ cKO. More broadly, our data surprisingly suggest that the linker sequence between EGF2 and LNS4 participates in organizing synapse morphology.

Although knockdown of $\operatorname{Nrxn} 3 \alpha$ did not elicit a functional synaptic phenotype (Fig. 2), we wanted to address whether the striking Nrxn3 $\alpha^{\text {A687T }}$ SS4- dependent increase in vGluT1 puncta size (Fig. $3 H$ ) could be simply explained as the ability of Nrxn3 $\alpha^{\text {A687T }}$ SS4-, but not Nrxn3 $\alpha$ WT SS4 - , to rescue a morphological phenotype induced by shRNA1. To address this potential explanation, we infected neurons with control shRNA or shRNA1 and quantified immunoreactive vGAT and vGluT1 cluster parameters. We found no differences in the density, intensity or size of vGAT or vGluT1 clusters (vGAT, density: Control: $3.26 \pm 0.22 ; n=44 / 3$; shRNA: $3.05 \pm 0.20 ; n=46 / 3 ; p=0.526$; intensity, Control: $51.1 \pm 9.96 \mathrm{AU}$; shRNA: $57.0 \pm 6.73 \mathrm{AU} ; p=$ 0.646; size, Control: $0.710 \pm 0.009 \mu \mathrm{m}^{2}$; shRNA: $0.680 \pm 0.043$ $\mu \mathrm{m}^{2} ; p=0.649$; vGluT1: density, Control: $4.16 \pm 0.074 ; n=$ 44/3; shRNA: $4.16 \pm 0.23 ; n=46 / 3 ; p=0.990$; intensity, Control: $55.5 \pm 10.4$ AU; shRNA: $55.8 \pm 8.87$ AU; $p=0.987$; size, Control: $0.994 \pm 0.089 \mu \mathrm{m}^{2}$; shRNA: $0.966 \pm 0.095 \mu \mathrm{m}^{2} ; p=0.839$; Student's $t$ test; Fig. $4 A-C)$. This is consistent with the notion that acute knockdown of endogenous $\operatorname{Nrxn} 3 \alpha$ alone does not alter synaptic properties (Fig. 2C-K) and reinforces the idea that the SS4- isoform of $\mathrm{Nrxn} 3 \alpha^{\mathrm{A} 687 \mathrm{~T}}$ selectively acts to robustly enhance vGluT1 cluster size.

\section{Molecular replacement with Nrxn $3 \alpha^{\mathrm{A687T}}$ SS4- selectively enhances excitatory presynaptic function in vitro}

Given the surprising morphological phenotype, we next asked whether this mutation alters basal inhibitory or excitatory synaptic transmission in primary hippocampal cultures. To study inhibitory transmission, we focused on the Nrxn3 $\alpha^{\text {A687T }} \mathrm{SS} 4+$ isoform because this isoform is highly expressed in subpopulations of GABAergic interneurons and displays the highest binding affinity to neuroligin-2, a key inhibitory postsynaptic neurexin ligand (Koehnke et al., 2010; Nguyen et al., 2016). We coinfected neurons with shRNA and Nrxn3 $\alpha^{\text {A687T }}$ SS4+ expressing lentiviruses at $4 \mathrm{DIV}$ and performed whole-cell electrophysiology at 13-14 DIV. Molecular replacement with WT or A687T Nrxn3 $\alpha$ did not alter passive membrane properties as membrane capacitance and resistance were unchanged $\left(C_{\mathrm{m}}\right.$ : shRNA: $145.8 \pm$ 
Postsynaptic Morphology - Molecular Replacement with Nrxn3a ${ }^{\mathrm{A} 687 \mathrm{~T}}$

A

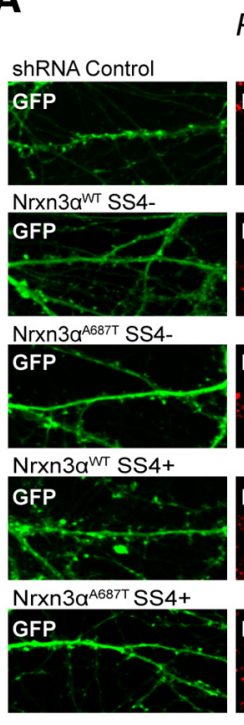

Postsynaptic Immunocytochemistry
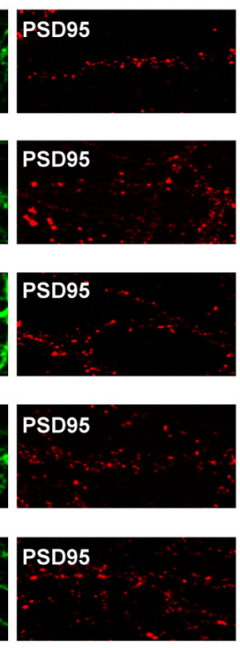
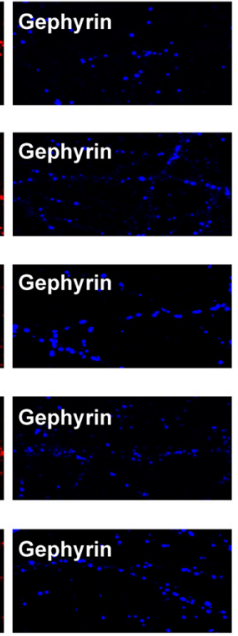
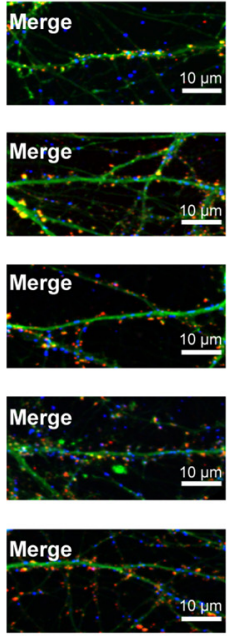

B
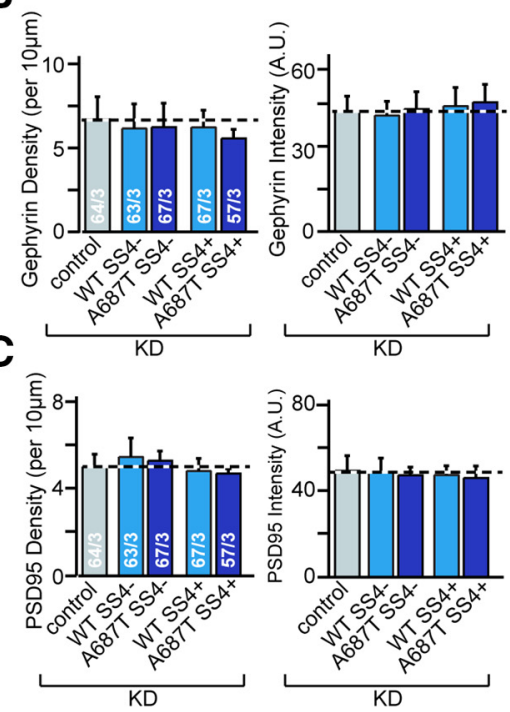
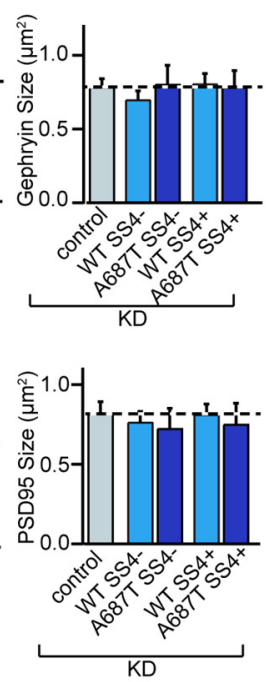

Presynaptic Morphology - Molecular Replacement with Nrxn3a ${ }^{\mathrm{A687T}}$

D

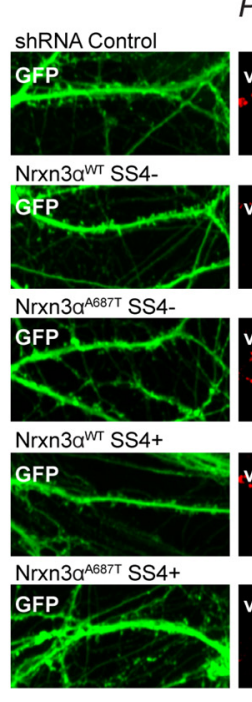

Presynaptic Immunocytochemistry
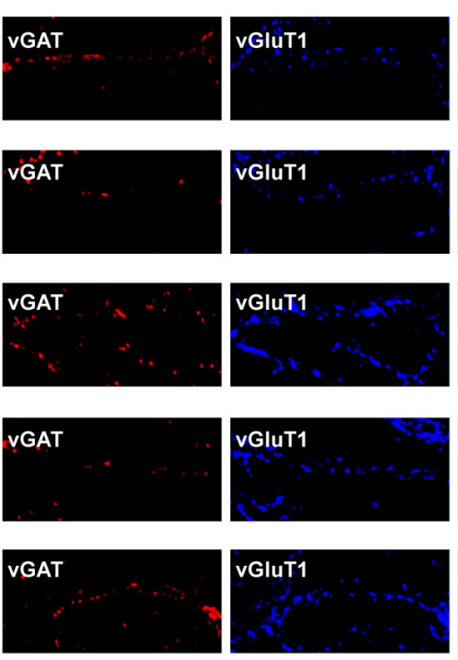
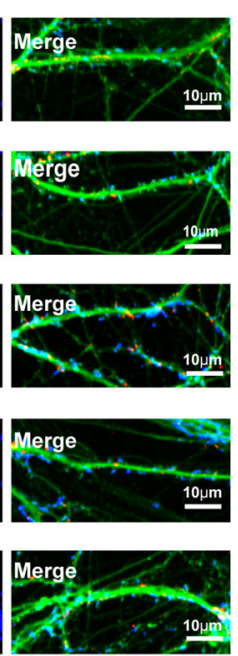

E
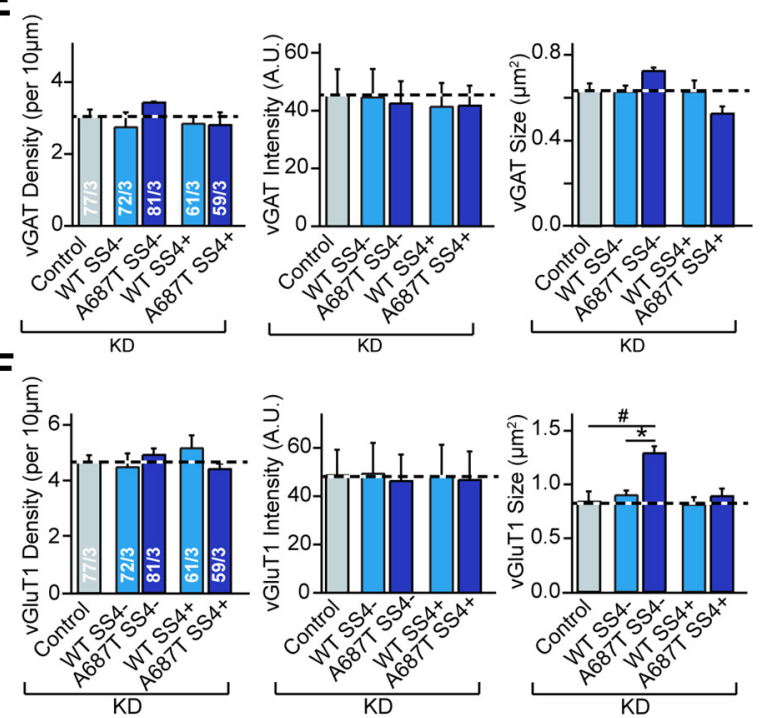

Figure 3. In vitro molecular replacement with Nrxn3 $\alpha^{\mathrm{A687T}} \mathrm{SS} 4-$ but not Nrxn3 $\alpha^{\mathrm{A687T}} \mathrm{SS} 4+$ selectively increases the size of excitatory presynaptic terminals. $\boldsymbol{A}$, Representative images. $\boldsymbol{B}, \boldsymbol{C}$, Summary graphs of inhibitory gephyrin (B) and excitatory PSD95 (C) postsynaptic immunoreactive cluster density, intensity and size reveal that excitatory and inhibitory postsynaptic morphology were not altered by either SS4 is oform of Nrxn3 $\alpha^{\text {A687T }}$. D-F, Same as $\boldsymbol{A}-\boldsymbol{C}$ except neurons were immunostained with antibodies to detect vGAT (inhibitory) and vGluT1 (excitatory) synaptic markers. Representative images of presynaptic vGAT and vGluT1-immunoreactive clusters $(\boldsymbol{D})$ and summary graphs of vGAT $(\boldsymbol{E})$ and vGluT1 (F) cluster density, intensity, and size reveal that Nrxn3 $\alpha$ A687T SS4 - selectively significantly increases vGluT1 cluster size. Data shown are mean \pm SEM; mean values calculated from the average of the number of total experiments. Numbers in bars represent cells/number of experiments. Statistical significance was determined by one-way ANOVA, multiple comparisons. ${ }^{*} p=0.05$, \#p $<0.05$ ).

$7.8369 \mathrm{pF}, n=45$; shRNA/WT SS4+: $153.1 \pm 7.598 \mathrm{pF}, n=59$; shRNA/A687T SS4+: $134.8 \pm 9.627 \mathrm{pF}, n=49, p=0.3358 ; R_{\mathrm{m}}$ : shRNA: $362.9 \pm 52.56 \mathrm{M} \Omega, n=45$; shRNA/WT SS4+: $346.1 \pm$ $25.11 \mathrm{M} \Omega, n=59$; shRNA/A687T SS4+:381.8 $\pm 35.66 \mathrm{M} \Omega, n=$ $49, p=0.8286$; one-way ANOVA, multiple comparisons; Fig. $5 A)$. Additionally, $\operatorname{Nrxn} 3 \alpha^{\mathrm{A} 687 \mathrm{~T}} \mathrm{SS} 4+$ did not induce changes in the frequency or amplitude of spontaneous mIPSCs (frequency, shRNA: $1.817 \pm 0.3052 \mathrm{~Hz}, n=45$; shRNA/WT SS4+: $1.657 \pm$ $0.3976 \mathrm{~Hz}, n=59$; shRNA/A687T SS4+: $1.648 \pm 0.202 \mathrm{~Hz}, n=$ 49, $p=0.9124$; amplitude, shRNA: $15.49 \pm 0.7477 \mathrm{pA}, n=45$; shRNA/WT SS4+: $16.45 \pm 1.737 \mathrm{pA}, n=59$; shRNA/A687T SS4+: $16.53 \pm 1.5131 \mathrm{pA}, n=49, p=0.8465$; one-way ANOVA, multiple comparisons' Fig. 5B). We next assessed actionpotential evoked IPSCs and found that the A687T mutation did not alter peak evoked IPSC amplitudes (shRNA: $1.806 \pm 0.3682$ $\mathrm{nA}, n=29$; shRNA/WT SS4+: $1.493 \pm 0.06414 \mathrm{nA}, n=26$; shRNA/A687T SS4+: $1.641 \pm 0.1688 \mathrm{nA}, n=23, p=0.6580$; one-way ANOVA, multiple comparisons; Fig. 5C). IPSC PPRs, an indirect measurement of presynaptic release, were also unchanged ( 40 ms ISI: shRNA: $0.5519 \pm 0.09024, n=29$; shRNA/WT SS4 $+: 0.4872 \pm 0.009555, n=26$; shRNA/A687T SS4+: $0.5103 \pm 0.08722, n=23, p=0.8535$; one-way ANOVA, multiple comparisons; Fig. $5 D$ ). Our electrophysiological analyses of inhibitory synaptic transmission are consistent with our 
A

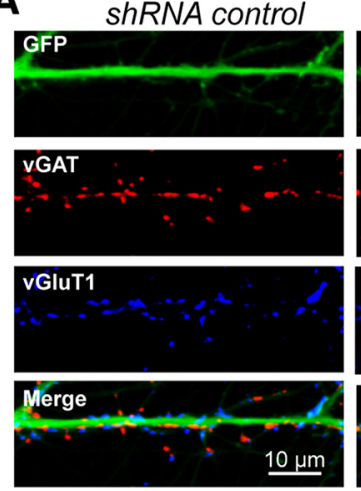

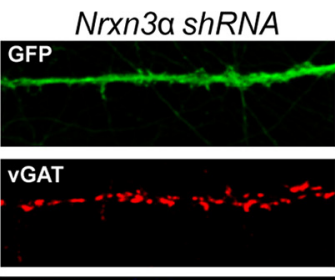

vGluT1

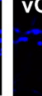

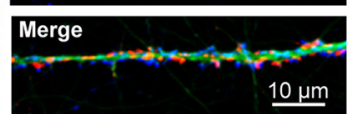

B

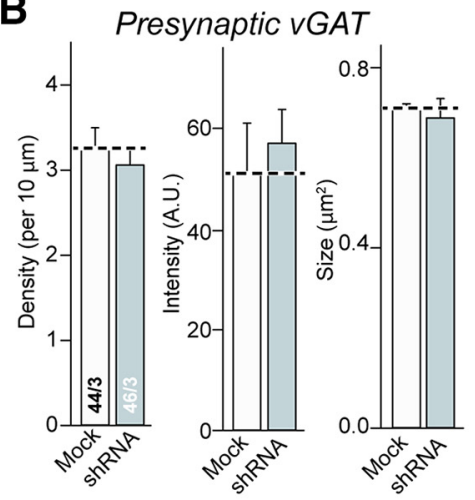

C

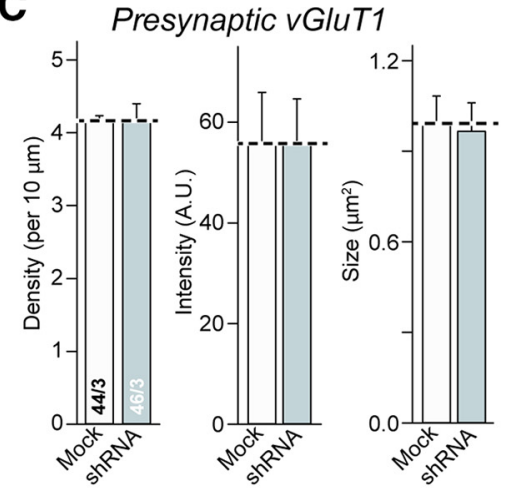

Figure 4. Knockdown of neurexin-3 $\alpha$ does not alter presynaptic morphology in cultured hippocampal neurons. $A$, Representative images of primary hippocampal neurons infected with shRNA or GFP control and immunostained with antibodies directed against inhibitory and excitatory presynaptic markers (vGAT and vGlut1). B, Summary graphs of vGAT-positive immunoreactive clusters properties. vGAT cluster density (left), cluster intensity (middle), and cluster size (right) were quantified. C, Summary graphs of vGluT1-positive immunoreactive clusters properties. vGluT1 cluster density (left), cluster intensity (middle), and cluster size (right) were quantified. Data shown are mean \pm SEM; mean values calculated from the average of the number of total experiments. $\boldsymbol{B}$, $\boldsymbol{C}$, Numbers in bars represent number of experiments. Statistical significance was determined by Student's $t$ test.

morphological analyses and argue that the A687T mutation does not alter inhibitory synapse function in primary hippocampal neurons.

We next measured excitatory synaptic transmission following molecular replacement with $\mathrm{Nrxn} 3 \alpha^{\mathrm{A} 687 \mathrm{~T}} \mathrm{SS} 4-$. We focused on the Nrxn $3 \alpha^{\mathrm{A} 687 \mathrm{~T}}$ SS4 - isoform because Nrxn3 $\alpha^{\mathrm{A} 687 \mathrm{~T}}$ SS4 - but not Nrxn3 $\alpha^{\text {A687T }}$ SS4+ selectively increased the size of presynaptic vGluT1 cluster size. In addition, Nrxn3 SS4- is highly expressed in excitatory neurons in hippocampus and plays a dominant role at excitatory hippocampal synapses to engage in functionally instructive transsynaptic interactions (Aoto et al., 2013, 2015; Fuccillo et al., 2015; Nguyen et al., 2016). We found that molecular replacement with Nrxn3 $\alpha^{\text {A687T }}$ SS4 - did not alter membrane capacitance or resistance (Fig. 5E). We first monitored spontaneous mEPSCs and observed a Nrxn $3 \alpha^{\mathrm{A} 687 \mathrm{~T}}$ SS4dependent increase in mEPSC frequency (shRNA: $1.08 \pm 0.11$ $\mathrm{Hz}, n=23 / 3$; shRNA/WT SS4-: $1.01 \pm 0.04 \mathrm{~Hz}, n=28 / 3$; shRNA/A687T SS4-: $1.92 \pm 0.28 \mathrm{~Hz}, n=31 / 3$; shRNA vs shRNA/MUT SS4 $-: p=0.033$; shRNA/WT SS4 - vs shRNA/ A687T SS4-: $p=0.236$; one-way ANOVA multiple comparisons) but not amplitude (shRNA: $11.7 \pm 0.93 \mathrm{pA}, n=23 / 3$; shRNA/WT SS4-: $10.8 \pm 0.48 \mathrm{pA}, n=28 / 3$; shRNA/A687T SS4-: $11.2 \pm 0.85 \mathrm{pA}, n=31 / 3$; shRNA vs shRNA/A687T SS4-: $p=0.90$; shRNA/WT SS4 - vs shRNA/A687T SS4 $-: p=0.91$; one-way ANOVA, multiple comparisons; Fig. $5 F$ ). mEPSC frequency is commonly thought to reflect changes in synapse numbers and/or changes in presynaptic release probability while mEPSC amplitudes typically correlate to changes in postsynaptic strength. Combined with our findings that molecular replacement with $\operatorname{Nrxn} 3 \alpha^{\mathrm{A} 687 \mathrm{~T}} \mathrm{SS} 4-$ does not alter excitatory synapse numbers, but rather vGluT1 cluster size (Fig. $3 H$ ), our functional data suggest that presynaptic properties are enhanced by molecular replacement with mutant $\operatorname{Nrxn} 3 \alpha$. To further test the consequence of $\operatorname{Nrxn} 3 \alpha^{\text {A687T }}$ SS4 - on synaptic transmission, we assessed electrically evoked AMPAR- and NMDAR-mediated excitatory postsynaptic synaptic currents. Consistent with the robust increase in mEPSC frequency (Fig. $5 F$ ), molecular replacement with $\operatorname{Nrxn} 3 \alpha^{\mathrm{A} 687 \mathrm{~T}}$ SS4 - induced a $\sim 50 \%$ increase in the amplitude of AMPAR- and NMDAR-mediated EPSCs (AMPAR, shRNA: $0.714 \pm 0.089 \mathrm{nA}, n=36 / 3$; shRNA/WT SS4 $-: 0.672 \pm$ $0.101 \mathrm{nA}, n=38 / 3$; shRNA/A687T SS4-: $1.464 \pm 0.058 \mathrm{nA}, n=$ 33/3; shRNA vs shRNA/A687T SS4-: $p=0.002$; shRNA/WT SS4- vs shRNA/A687T SS4-: $p=0.001$; NMDAR, shRNA:
$0.488 \pm 0.076 \mathrm{nA}, n=38 / 3 ;$ shRNA/WT SS4-: $0.411 \pm 0.109$ $\mathrm{nA}, n=42 / 3$; shRNA/A687T SS4 $-: 0.909 \pm 0.127 \mathrm{pA}, n=39 / 3$; shRNA vs shRNA/A687T SS4-: $p=0.047$; shRNA/WT SS4 - vs shRNA/A687T SS4-: $p=0.0375$; one-way ANOVA, multiple comparisons; Fig. 5G,H). To quantify presynaptic release, we monitored NMDAR-mediated evoked EPSC PPRs at increasing ISIs. PPRs provide sensitive, but indirect measurements of presynaptic release. We found that replacement with the splice-site 4 lacking isoform of $\operatorname{Nrxn} 3 \alpha^{\mathrm{A} 687 \mathrm{~T}}$ significantly reduced the NMDAR PPR, which is consistent with an increase in presynaptic release probability ( $40 \mathrm{~ms}$ ISI, shRNA: $0.721 \pm 0.055, n=24$; shRNA/WT SS4 $+: 0.760 \pm 0.062, n=23$; shRNA/A687T SS4+: $0.573 \pm 0.0618, n=28$; shRNA vs shRNA/A687T SS4-: $p=$ 0.284; shRNA/WT SS4 - vs shRNA/A687T SS4-: $p=0.015$; one-way ANOVA, multiple comparisons; Fig. 5I).

Given the striking SS4- dependent effect on presynaptic vGluT1 cluster size and the unexpected increase in mEPSC frequency, AMPAR- and NMDAR-mediated evoked EPSC amplitudes, and presynaptic release probability, we next asked whether Nrxn3 $\alpha^{\mathrm{A} 687 \mathrm{~T}}$ SS4 + could induce an identical synaptic phenotype. Similar to molecular replacement with $\mathrm{Nrxn} 3 \alpha^{\mathrm{A} 687 \mathrm{~T}}$ SS4-, Nrxn3 $\alpha^{\mathrm{A} 687 \mathrm{~T}} \mathrm{SS} 4+$ did not alter membrane capacitance or resistance $\left(C_{\mathrm{m}}\right.$, shRNA: $164.2 \pm 8.96 \mathrm{pF}, n=19$; shRNA/WT SS4+: $161.0 \pm 8.9 \mathrm{pF}, n=22$; shRNA/A687T SS4+: $166.1 \pm 6.47 \mathrm{pF}$, $n=23$; shRNA vs shRNA/A687T SS4 $+: p=0.987$; shRNA/WT SS4+ vs shRNA/A687T SS4+: $p=0.900 ; R_{\mathrm{m}}$, shRNA: $180.7 \pm$ $12.9 \mathrm{M} \Omega, n=17$; shRNA/WT SS4+: $191.7 \pm 10.4 \mathrm{M} \Omega, n=20$; shRNA/A687T SS4+: $193.8 \pm 12.2 \mathrm{M} \Omega, n=17$; shRNA vs shRNA/A687T SS4+: $p=0.723$; shRNA/WT SS4 + vs shRNA/ A687T SS4+: $p=0.991$; one-way ANOVA, multiple comparisons; Fig. 6A). However, unlike molecular replacement with Nrxn3 $\alpha^{\text {A687T }}$ SS4 -, Nrxn3 $\alpha^{\text {A687T }}$ SS4 + did not impact mEPSC frequency (shRNA: $2.06 \pm 0.73 \mathrm{~Hz}, n=19 / 3$; shRNA/WT SS4+: $2.44 \pm 0.63 \mathrm{~Hz}, n=22 / 3$; shRNA/A687T SS4 $+: 1.99 \pm 0.38 \mathrm{~Hz}$, $n=23 / 3$; shRNA vs shRNA/A687T SS4 $+: p=0.996 ;$ shRNA/WT SS4+ vs shRNA/A687T SS4+: $p=0.863$; one-way ANOVA, multiple comparisons) or amplitude (shRNA: $15.3 \pm 0.35 \mathrm{pA}$, $n=19 / 3$; shRNA/WT SS4 $+: 15.2 \pm 1.78 \mathrm{pA}, n=22 / 3$; shRNA/ A687T SS4+: $15.0 \pm 1.72 \mathrm{pA}, n=23 / 3$; shRNA vs shRNA/A687T SS4+: $p=0.984 ;$ shRNA/WT SS4+ vs shRNA/A687T SS4+: $p=0.999$; one-way ANOVA, multiple comparisons), evoked AMPAR-mediated (shRNA: $0.4955 \pm 0.0607 \mathrm{nA}, n=32, p=$ 0.7994 ; shRNA/WT SS4 $+: 0.5394 \pm 0.08129 \mathrm{nA}, n=29$; shRNA/ 


\section{A} Passive Membrane Properties

\section{$B$}
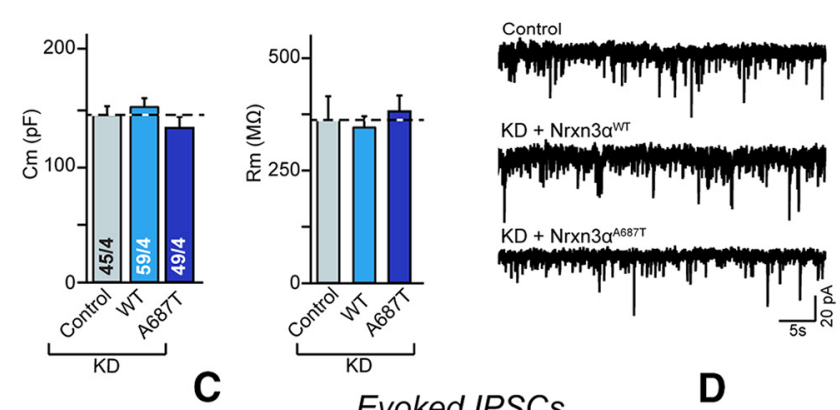

D
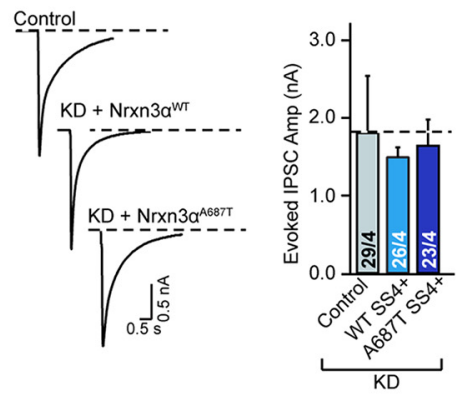

Miniature Inhibitory Postsynaptic Currents
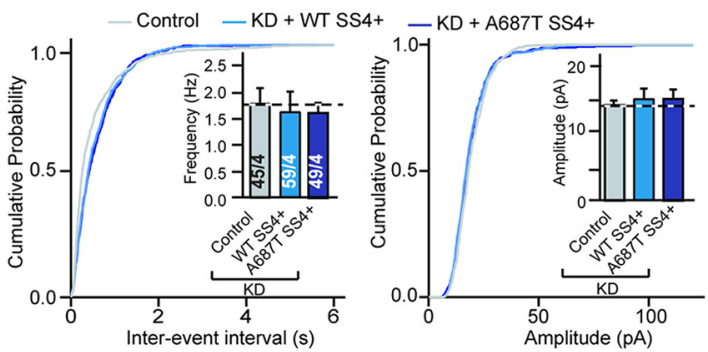

IPSC Paired-pulse Ratios

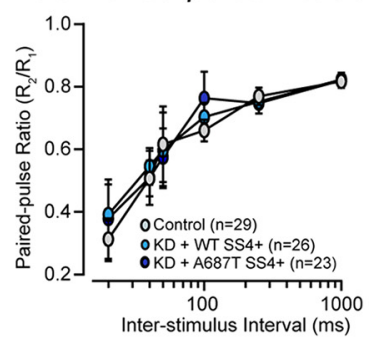

\section{Excitatory synaptic transmission - Molecular Replacement with Nrxn3a ${ }^{\mathrm{A687T}}$ SS4-}

\section{E Passive Membrane Properties F}

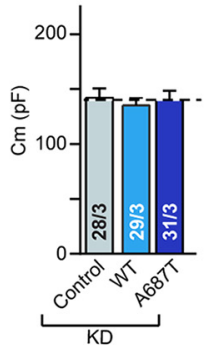

G

Evoked AMPAR EPSCs

Control

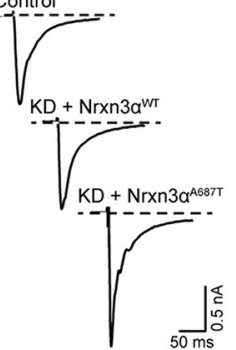

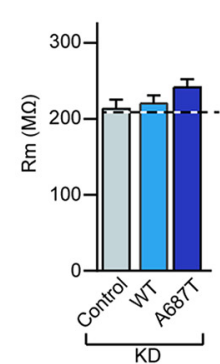

H

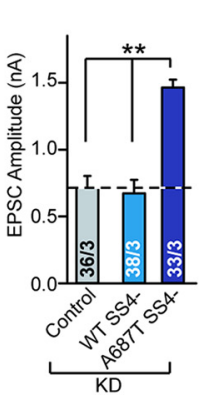

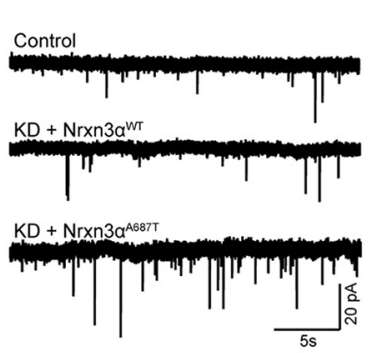

Evoked NMDAR EPSCs

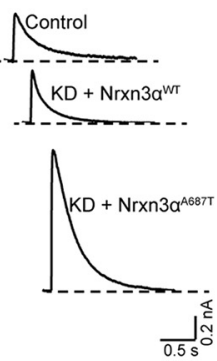

Miniature Excitatory Postsynaptic Currents
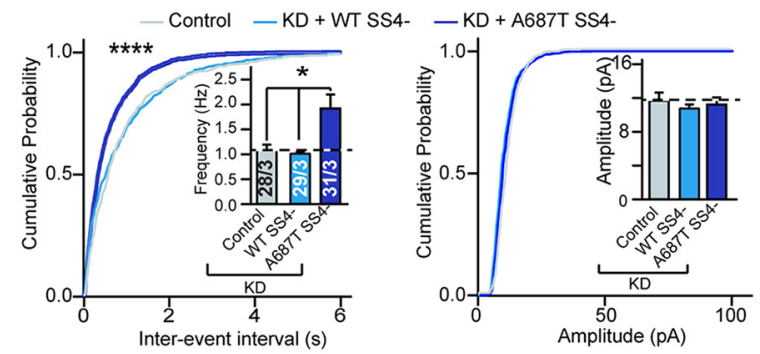

I

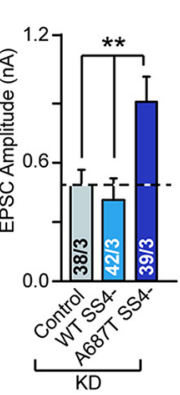

NMDAR Paired-pulse Ratios

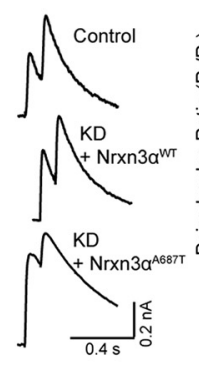

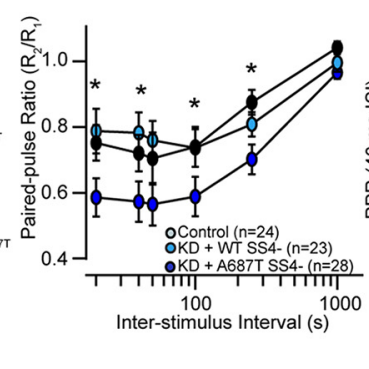

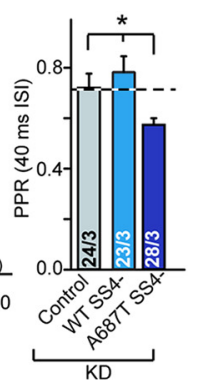

Figure 5. Molecular replacement with Nrxn3 $\alpha^{\mathrm{A687T}}$ SS4 - enhances presynaptic release probability at excitatory synapses. $\boldsymbol{A}-\boldsymbol{D}$, Molecular replacement with WT or A687T Nrxn3 $\alpha$ SS4 + does not alter inhibitory synaptic transmission in cultured hippocampal neurons. $\boldsymbol{A}$, Summary graph of passive membrane properties in control neurons or following molecular replacement with Nrxn3 $\alpha^{\text {WT }}$ or Nrxn3 $\alpha{ }^{\text {A687T } S S 4+. ~ M e m b r a n e ~ c a p a c i t a n c e ~}\left(C_{m}\right)$ and membrane resistance $\left(R_{\mathrm{m}}\right)$ were unchanged between conditions. $\boldsymbol{B}$, Nrxn3 $\alpha{ }^{\text {A687T }}$ SS4 + does not change mIPSC amplitudes or

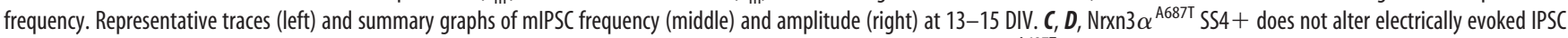
amplitudes $(\boldsymbol{C})$ or PPRs $(\boldsymbol{D})$, representative traces (left) and summary graph (right). $\boldsymbol{E}-\boldsymbol{I}$, Molecular replacement with Nrxn3 $\alpha^{\text {A687T }}$ SS4- significantly increases presynaptic release probability at

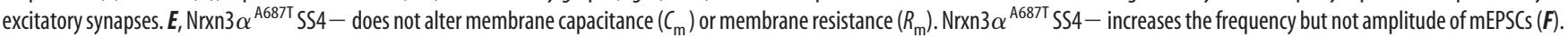
Representative traces (left) and summary graphs of mEPSC frequency (middle) and amplitude (right) recorded from 13-15 DIV primary hippocampal neurons. The SS4- isoform of Nrxn3 $\alpha$ A687T significantly increased electrically evoked AMPAR-mediated $(\boldsymbol{G})$ and NMDAR-EPSC amplitudes ( $\boldsymbol{H}$ ). Representative traces (left) and summary graph (right) for evoked AMPAR-and NMDAR-mediated

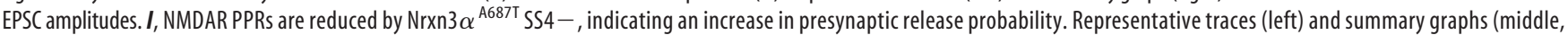
right) of NMDAR EPSC PPRs at 13-15 DIV. Data shown are mean \pm SEM; mean values calculated from the average of the number of total experiments. Numbers in bars represent cells/number of experiments. Statistical significance was determined by one-way ANOVA, multiple comparisons. ${ }^{*} p<0.05,{ }^{* *} p<0.01,{ }^{* * * *} p<0.0001$. 


\section{Excitatory synaptic transmission - Molecular Replacement with Nrxn3a ${ }^{\mathrm{A687T}} \mathrm{SS} 4+$}

\section{A Passive Membrane Properties}
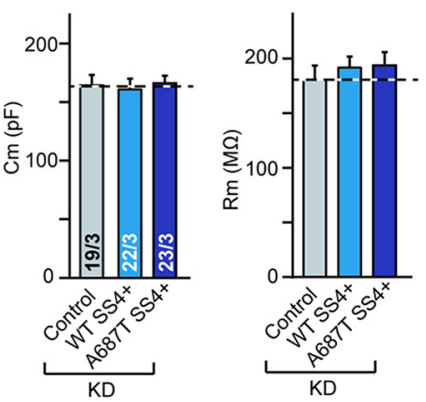

C Evoked AMPAR EPSCs

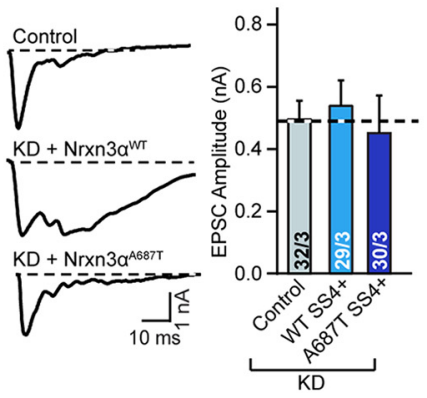

B

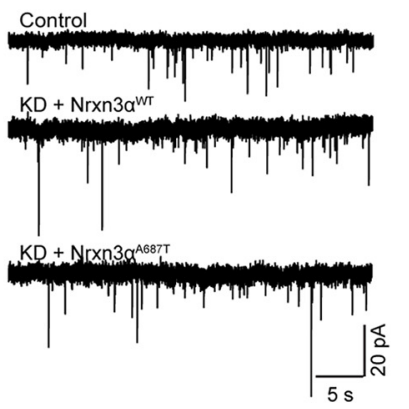

D Evoked NMDAR EPSCs

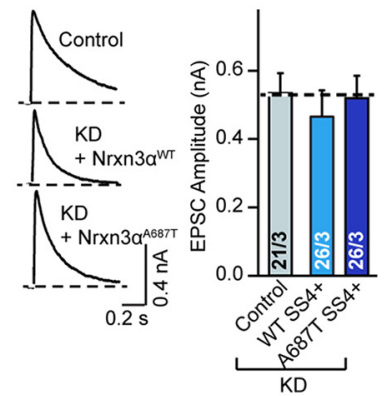

mEPSCs

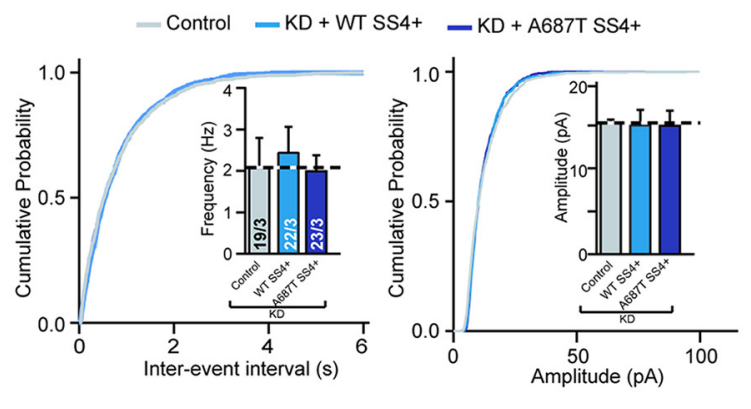

E

NMDAR Paired-pulse Ratios

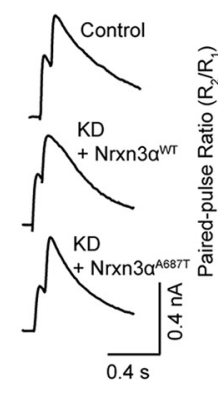

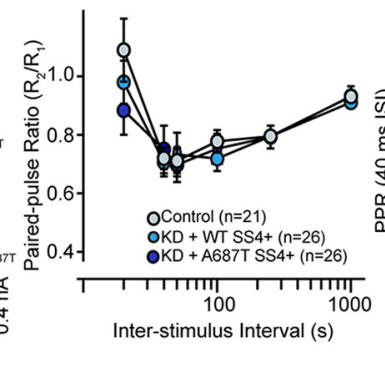

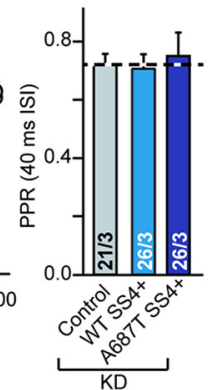

Figure 6. Molecular replacement with Nrxn3 $\alpha^{\mathrm{A687T}} \mathrm{SS} 4+$ has no effect on excitatory transmission. $\boldsymbol{A}-\boldsymbol{E}$, Same as Figure $5 E-\boldsymbol{I}_{\text {, except Nrxn3 } \alpha}{ }^{\mathrm{A687T}}$ SS4 + was used for molecular replacement. $A$, Summary graph of passive membrane properties in control neurons or following molecular replacement with $S S 4+N$ rxn $3 \alpha{ }^{\text {WT }}$ or Nrxn3 $\alpha^{\text {A687T }}$. Membrane capacitance $\left(C_{m}\right)$ and membrane resistance $\left(R_{\mathrm{m}}\right)$ were unchanged between conditions. $\boldsymbol{B}-\boldsymbol{E}$, The $S S 4+$ Nrxn3 $\alpha$ isoform did not alter excitatory synaptic transmission properties. $\boldsymbol{B}$, mEPSC frequency and amplitudes are unchanged. Representative traces (left) and summary graphs of mEPSC frequency (middle) and amplitude (right). C, D, Nrxn3 $\alpha^{\text {A687T }}$ SS4+ does not alter electrically evoked AMPAR-mediated (C) or NMDAR-mediated (D) synaptic transmission. Representative traces (left) and summary graph (right) at 13-15 DIV. E, Molecular replacement with Nrxn3 $\alpha^{\text {A687T }}$ SS4 + does not change presynaptic release. Representative traces (left) and summary graphs (middle and right) of NMDAR EPSC PPRs at 13-15 DIV. Data shown are mean \pm SEM; mean values calculated from the average of the number of total experiments. Numbers in bars represent cells/number of experiments. Statistical significance was determined by one-way ANOVA, multiple comparisons.

A687T SS4+: $0.4519 \pm 0.1204 \mathrm{nA}, n=30)$, NMDAR-mediated EPSC amplitudes (shRNA: $0.5346 \pm 0.0586 \mathrm{nA}, n=21, p=$ 0.7497; shRNA/WT SS4+: $0.4659 \pm 0.3058 \mathrm{nA}, n=26$; shRNA/ A687T SS4+: $0.5204 \pm 0.06491 \mathrm{nA}, n=26)$, or NMDAR PPRs (40 ms ISI, shRNA: $0.7205 \pm 0.03718, n=21$;shRNA/WT SS4+: $0.707 \pm 0.04925, n=26$; shRNA/A687T SS4+: $0.7501 \pm$ $0.08145, n=26, p=0.8733$; one-way ANOVA, multiple comparisons; Fig. $6 B-E$ ). Together, these data suggest that the A687T mutation induces a SS4-dependent gain-of-function phenotype by selectively increasing presynaptic efficacy at excitatory synapses, which provides crucial insight into this extracellular sequence.

\footnotetext{
Nrxn $3 \alpha^{\mathrm{A} 687 \mathrm{~T}}$ SS4- increases readily releasable pool size at excitatory synapses and the number of docked vesicles Presynaptically, action potential-evoked excitatory transmission is a function of the likelihood that a single vesicle of neurotransmitter will be exocytosed following an action potential and the number of vesicles ready to release (Kaeser and Regehr, 2017). Therefore, in addition to the decreased PPRs following molecular replacement with $\operatorname{Nrxn} 3 \alpha^{\text {A687T }}$ SS4-, changes in the size of the readily releasable pool (RRP) might contribute to the excitatory transmission phenotype (Fig. $5 F$ ) and electrically evoked AMPARand NMDAR-mediated EPSC amplitudes (Figs. 5G,H). We analyzed the size of the RRP using two distinct approaches. We functionally measured the size of the RRP using $0.5 \mathrm{M}$ hypertonic
}

sucrose and performed electron microscopy (EM) on cultured neurons to quantify the number of docked vesicles.

First, we monitored IPSCs and AMPAR-mediated EPSCs evoked by the acute $(30 \mathrm{~s})$ application of $0.5 \mathrm{M}$ hypertonic sucrose (Kaeser and Regehr, 2017). We found that the size of the inhibitory RRP was not altered by $\operatorname{Nrxn} 3 \alpha^{\mathrm{A} 687 \mathrm{~T}}$ relative to control and Nrxn $3 \alpha$ WT SS4 - conditions (shRNA: $12.863 \pm 3.212 \mathrm{nC}, n=$ 29; shRNA/WT SS4+: $11.573 \pm 3.322 \mathrm{nC}, n=27$; shRNA/A687T $\mathrm{SS} 4+: 12.895 \pm 5.188 \mathrm{nC}, n=24, p=0.9654)$, which is consistent with the findings that $\operatorname{Nrxn} 3 \alpha^{\text {A687T }}$ does not alter inhibitory morphology or synaptic function (Fig. 7A). By contrast, molecular replacement with $\mathrm{Nrxn} 3 \alpha^{\mathrm{A} 687 \mathrm{~T}} \mathrm{SS} 4-$ increased EPSC charge transfer, which is indicative of an increase in the size of the RRP ( $\operatorname{shRNA:~} 4.51 \pm 0.29 \mathrm{nC}, n=24 / 3$; shRNA/WT SS4-: $4.31 \pm$ $0.45 \mathrm{nC}, n=29 / 3$; shRNA/A687T SS4-: $7.25 \pm 0.62 \mathrm{nC}, n=$ 38/3; shRNA vs shRNA/A687T SS4-: $p=0.0148$; shRNA/WT SS4- vs shRNA/A687T SS4-: $p=0.0107$; one-way ANOVA multiple comparisons; Fig. $7 B$ ). Together, the surprising increase in presynaptic efficacy induced by $\operatorname{Nrxn} 3 \alpha^{\mathrm{A} 687 \mathrm{~T}} \mathrm{SS} 4-$ is a consequence of an increase in both presynaptic release probability and size of the functional RRP.

Second, we quantified the impact of $\operatorname{Nrxn} 3 \alpha^{\mathrm{A} 687 \mathrm{~T}}$ on the ultrastructure of hippocampal synapses in culture using EM. Similar to our electrophysiological analyses, we coinfected primary hippocampal cultures at 4 DIV with lentiviruses to perform molecular replacement with SS4 isoforms of $\mathrm{Nrxn} 3 \alpha$ and processed 
the neurons at 14 DIV for EM. We measured AZ length, the number of docked vesicles $\leq 100 \mathrm{~nm}$ from the $\mathrm{AZ}$ and the number of vesicles per $\mathrm{AZ}$ area from all analyzable synapses. In primary culture, the majority of neurons are excitatory (Benson et al., 1994) and excitatory synapses represent $65-75 \%$ of all dendritic synapses (Benson and Cohen, 1996), thus, the majority of synapses analyzed likely represent excitatory synapses. Consistent with this and in agreement with our electrophysiological assessment, we found that although the length of the AZ did not change, molecular replacement with Nrxn3 $\alpha^{\text {A687T }}$ SS4 - significantly increased the number of docked vesicles per $A Z$ length and the number of vesicles per AZ area (putative AZ length, shRNA: $0.2667 \pm 0.01022 \mu \mathrm{m}, n=81$; shRNA/WT SS4-: $0.2886 \pm 0.01148 \mu \mathrm{m}$, $n=69$; shRNA/A687T SS4-: $0.2492 \pm$ $0.01245 \mu \mathrm{m}, n=56, p=0.0633$; docked vesicles/100 nm of putative AZ, shRNA: $1.466 \pm 0.07305, n=81 ;$ shRNA/WT SS4-: $1.522 \pm 0.08064, n=70$; shRNA/ A687T SS4-: $1.869 \pm 0.094, n=59$; shRNA vs shRNA/A687T SS4-: $p=$ 0.0019 ; shRNA/WT SS4- vs shRNA/ A687T SS4-: $p=0.0123$; vesicles per putative AZ area, shRNA: $0.4024 \pm 0.0139$, $n=82$; shRNA/WT SS4-: $0.3941 \pm$ $0.01833, n=70$; shRNA/A687T SS4-: $0.4559 \pm 0.01821, n=59 ;$ shRNA vs shRNA/A687T SS4-: $p=0.0481$; shRNA/ WT SS4- vs shRNA/A687T SS4-: $p=$ 0.0234; one-way ANOVA multiple comparisons; Fig. 7C). Our ultrastructural examination of excitatory and inhibitory synapses validates our electrophysiological analyses, indicating that one role for the sequences between EGF2 and LNS4 is to regulate RRP size at excitatory synapses. We next asked whether the Nrxn3 $\alpha^{\text {A687T }}$ SS4- gain-of-function presynaptic phenotype identified in developing primary hippocampal neurons is also present in more mature neurons within an intact circuit.

In vivo presynaptic molecular replacement with $\operatorname{Nrxn} 3 \alpha^{\mathrm{A687T}}$ increases basal excitatory presynaptic properties and impairs presynaptic LTP in ex vivo slices

We next performed molecular replacement in vivo and measured synaptic transmission in ex vivo acute hippocampal slices at CA1subiculum synapses. We stereotactically coinjected P21 mice in CA1 with an AAV encoding Nrxn $3 \alpha$ shRNA and mRuby, and an AAV encoding WT or A687T Nrxn3 $\alpha$ SS4 - (Fig. 8A). Three to 4 weeks after injection, we prepared $300 \mu \mathrm{m}$ horizontal slices and measured excitatory synaptic transmission at CA1-subiculum synapses (Aoto et al., 2013). We focused on this circuit because we can largely restrict infection to presynaptic CA1 neurons and monitor excitatory synaptic strength from uninfected postsynaptic subicular principle neurons to unambiguously study the presynaptic contribution of $\operatorname{Nrxn} 3 \alpha$ (Fig. 8B,C; Aoto et al., 2013,

C
B
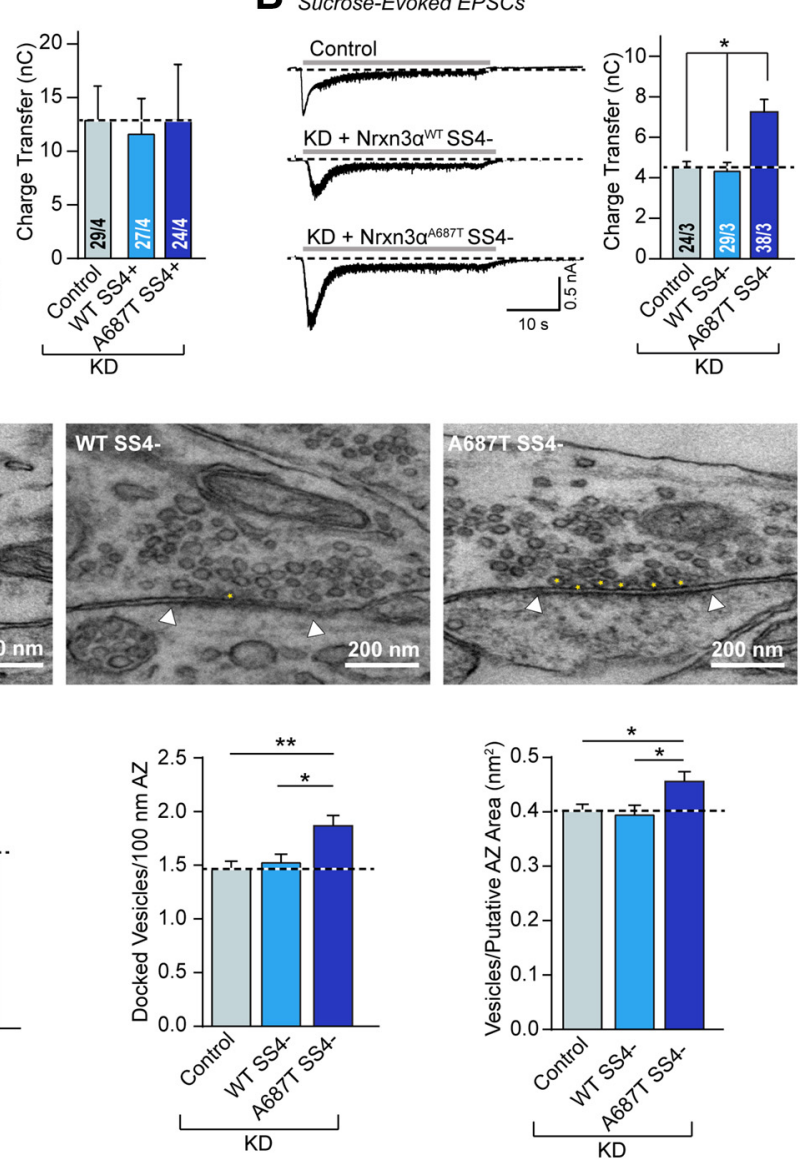

Figure 7. Nrxn3 $\alpha^{\text {A687T }}$ SS4 - increases the size of the RRP, total number of vesicles and the number of docked vesicles at (left) and summary graph right for with shRNA (control) or coinfected with shRNA and Nrxn3 $\alpha^{\text {WT }}$ or Nrxn3 $\alpha^{\text {A687T }}$ SS4 - conditions. B, Quantification of the RRP at excitatory synapses following acute application of hypertonic sucrose $(0.5 \mathrm{~m})$. Representative traces (left) and summary graph right for with shRNA (control) or coinfected with shRNA

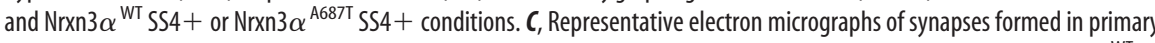
hippocampal cultures following lentiviral infection with shRNA alone (control) or coinfection with shRNA and Nrxn3 $\alpha^{\text {WT }}$ or SS4 - (top). Summary graphs of micrographs (bottom) of putative AZ length (left), number of docked vesicles pe end of putative PSD, and yellow stars mark docked vesicles. Data shown are mean \pm SEM; mean values calculated from the average of the number of synapses analyzed. Numbers in bars represent synapses/number of independent experiments. Statistical significance was determined by one-way ANOVA, multiple comparisons. ${ }^{*} p<0.05,{ }^{* *} p<0.001$.

2015; Anderson et al., 2015; Dai et al., 2019). To stimulate CA1 afferents, we placed an extracellular stimulating electrode in the stratum oriens/alveus and recorded from two major types of principle neurons in subiculum, identified by action-potential spiking patterns as regular-spiking (RS) or burst-spiking (BS; Fig. $8 C)$. Presynaptic Nrxn3 $\alpha^{\text {A687T }}$ SS4 - dramatically enhanced the AMPAR input/output relationship (I/O slope RS, shRNA $1.692 \pm 0.2417, n=28$; shRNA/WT SS4 $-1.444 \pm 0.2257, n=$ $18, p=0.0059$; shRNA/ A687T SS4- $3.288 \pm 0.5766, n=19$; shRNA vs shRNA/A687T SS4-: $p=0.0059 ;$ shRNA/WT SS4- vs shRNA/A687T SS4-: $p=0.0039$, Fig. $8 D$; I/O slope BS, shRNA $1.515 \pm 0.2386, n=35$; shRNA/WT SS4 $-1.525 \pm 0.3146, n=$ 21; shRNA/A687T SS4 - $3.27 \pm 0.4437, n=21$; shRNA vs shRNA/A687T SS4-: $p=0.0005$; shRNA/WT SS4 - vs shRNA/ A687T SS4-: $p=0.0022$; one-way ANOVA, multiple comparisons; Fig. $8 D, E$ ). Additionally we observed decreased AMPARmediated PPRs at CA1-RS and CA1-BS synapses (R2/R1 RS, shRNA $1.748 \pm 0.07283 ; n=36 ;$ shRNA/WT - SS4 $1.834 \pm$ $0.0904, n=30$; shRNA/A687T -SS4, $n=39$; shRNA ns shRNA/ 
A AAV Stereotactic Injection into CA1

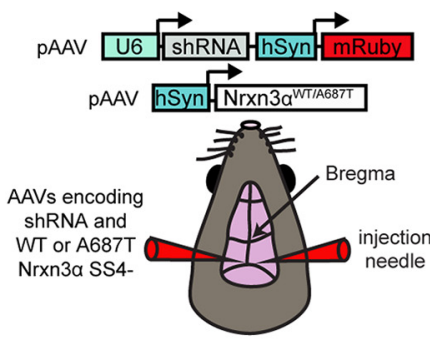

Dorsal View

\section{D}

CA1 - Regular Spiking Cell AMPAR Input-Output
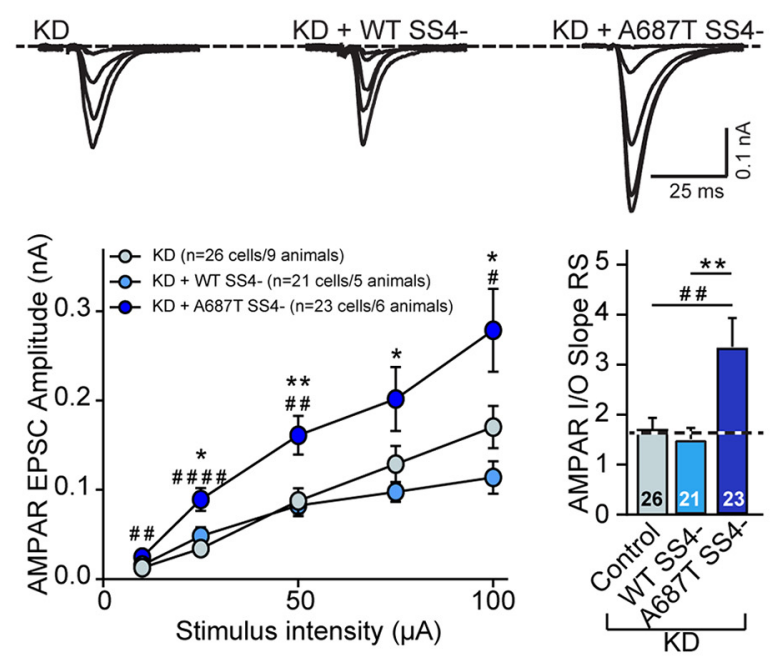

$\mathbf{F}$

CA1-Subiculum Regular Spiking Cell PPR (40 ms ISI)
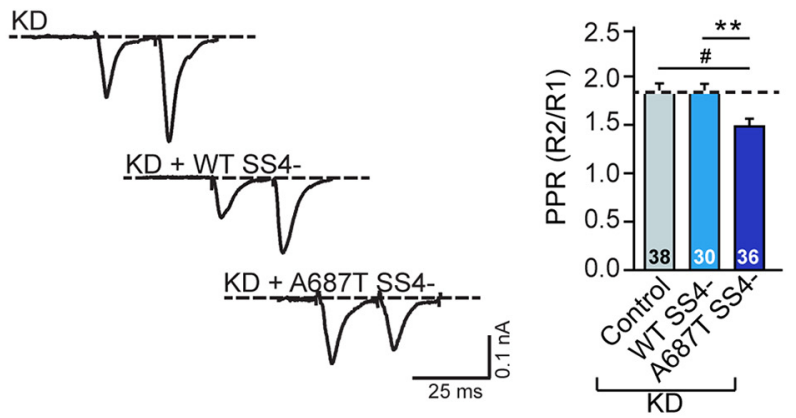

B $_{\text {Recording Scheme }}$

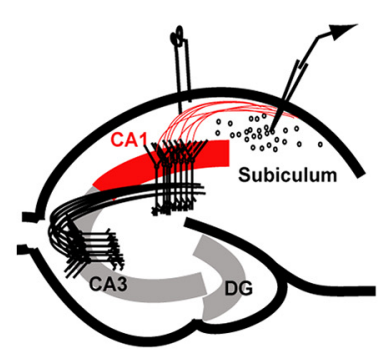

E

CA1 - Burst Spiking Cell AMPAR Input-Output
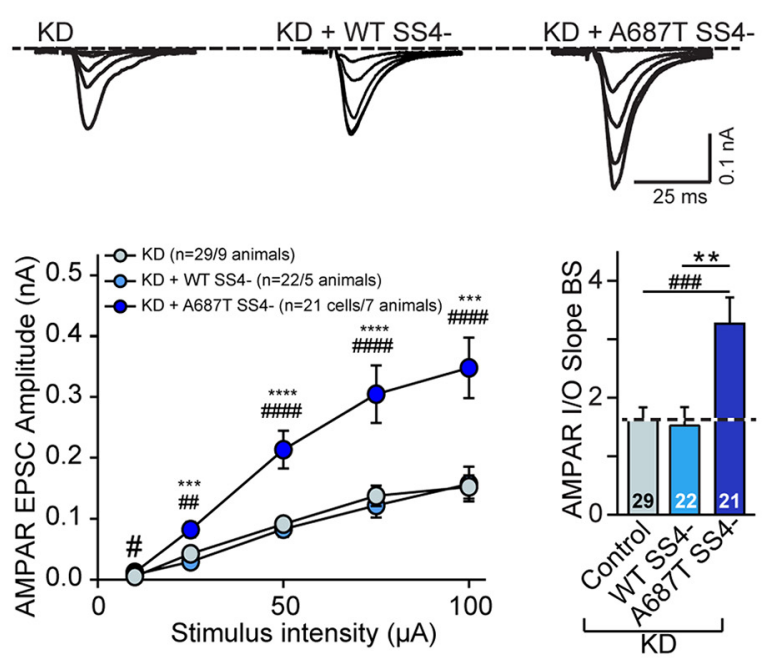

$\mathbf{G}_{\text {CA1-Subiculum Burst Spiking Cell PPR (40ms ISI) }}$
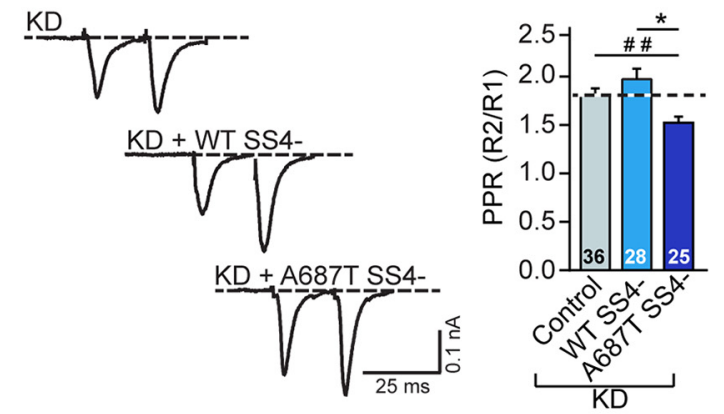

Figure 8. Presynaptic Nrxn3 $\alpha^{\mathrm{A687T}} \mathrm{SS} 4$ - expression in vivo enhances basal synaptic transmission at CA1-subiculum synapses in ex vivo acute hippocampal slices. $A$, Coinjection strategy with AAVs encoding Nrxn3 $\boldsymbol{\alpha}$ shRNA and Nrxn3 $\alpha^{\text {WT }}$ or Nrxn3 $\alpha^{\text {A687T } S S 4-~(l e f t) ~ a n d ~ r e p r e s e n t a t i v e ~ i m a g e ~ o f ~} 100 \mu \mathrm{m}$ horizontal slice of hippocampus showing specific infection of CA1 (right). $\boldsymbol{B}$, Recording scheme to isolate infected CA1 afferents and measure electrically evoked EPSCs from uninfected regular and burst spiking neurons in subiculum. C, Representative traces of RS and BS subicular neurons. $\boldsymbol{D}, \boldsymbol{E}$, Presynaptic molecular replacement with Nrxn3 $\alpha^{\mathrm{A} 687 \mathrm{~T}} \mathrm{SS4}$ - significantly enhances the AMPAR-mediated input-output relationship for both regular- and burst-spiking neurons. AMPAR input/output representative traces (top), summary graph (left), and slope (right) for RS (D) and BS (E) neurons. F, G, Presynaptic molecular replacement with Nrxn3 $\alpha^{\text {A687T } S S 4-}$ significantly reduces AMPAR-mediated PPRs, indicating an increase in presynaptic release probability. Representative traces (left) and summary graph (right) of AMPAR PPR for RS (F) and BS (G) neurons. Data shown are mean $\pm \mathrm{SEM}$; mean values calculated from the number of cells analyzed. Numbers in bars represent the number of animals used for each experiment. Statistical significance was determined by one-way ANOVA, multiple comparisons. ${ }^{*} p<0.05,{ }^{* *} p<0.01,{ }^{* * *} p<0.001$, ${ }^{* * * *} p<0.0001$, \#p $<0.05$, \#\#p $<0.01$, \#\#\#p $<0.001$, \#\#\#\#p $<0.0001$.

A687T SS4-: $p=0.0458 ;$ shRNA/WT SS4 - vs shRNA/A687T SS4-: $p=0.0081$; Fig. $8 F$; R2/R1 BS, shRNA $1.81 \pm 0.06886, n=$ 36; shRNA/WT SS4- $1.973 \pm 0.1092, n=30$; shRNA/A687T SS4 $-1.525 \pm 0.06085, n=26$; shRNA vs shRNA/A687T SS4-: $p=0.0466$; shRNA/WT SS4 - vs shRNA/A687T SS4-: $p=$ 0.0015; one-way ANOVA, multiple comparisons; Fig. $8 F, G)$. These results from acute hippocampal slices are congruent with the presynaptic phenotype observed in primary neurons and support the idea that the A687T mutation in $\mathrm{Nrxn} 3 \alpha$
SS4- causes a selective increase in basal presynaptic efficacy at excitatory synapses. We next asked whether presynaptic Nrxn3 $\alpha^{\text {A687T }}$ SS4- alters activity-dependent synaptic plasticity.

The CA1-subiculum circuit is advantageous to study the function of presynaptic molecules for two major reasons. First, as discussed above, it is easy to selectively target presynaptic CA1 neurons with minimal spillover to subiculum to specifically study presynaptic function. Second, CA1 afferents onto regular and 
A

CA1-Subiculum Regular Spiking Cell LTP
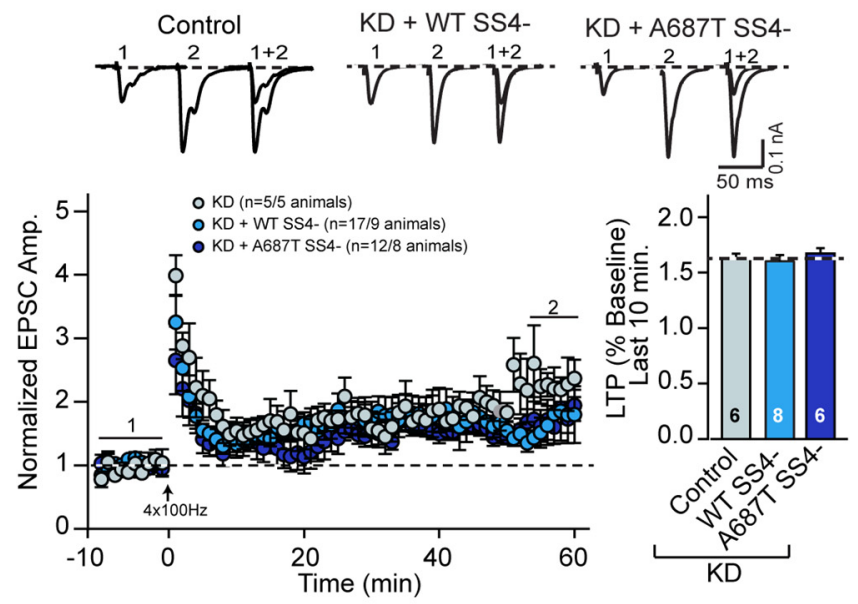

C CA1-Subiculum Regular-spiking PPR Pre- vs Post-LTP Control
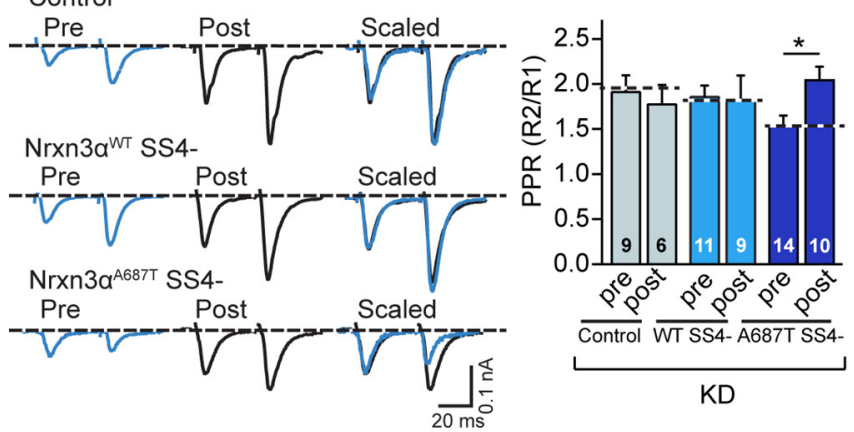

B

CA1-Subiculum Burst Spiking cell LTP
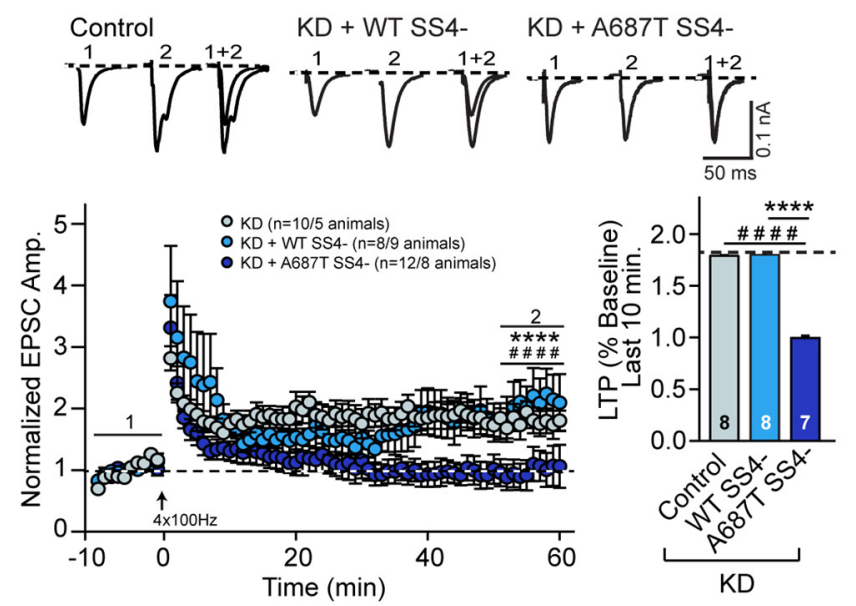

D CA1-Subiculum Burst Spiking PPR Pre- vs Post-LTP
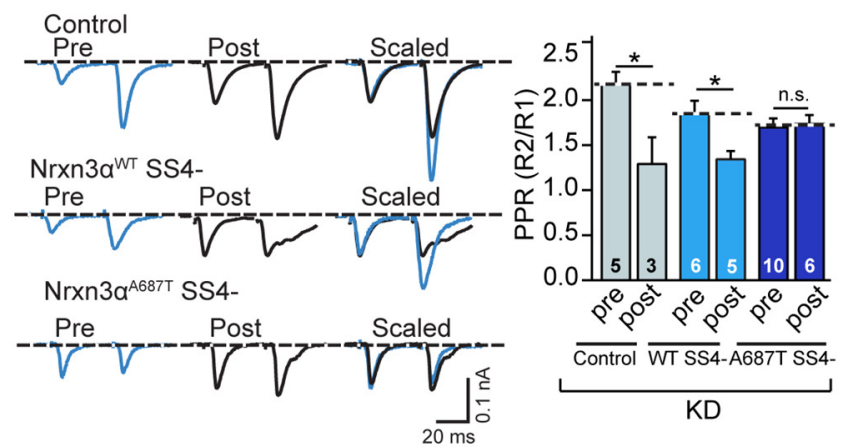

Figure 9. Presynaptic Nrxn3 $\alpha^{\text {A687T } S S 4-~ e x p r e s s i o n ~ i m p a i r s ~ p r e s y n a p t i c ~ b u t ~ n o t ~ p o s t s y n a p t i c ~ L T P a t ~ C A 1-s u b i c u l u m ~ s y n a p s e s ~ i n ~ e x ~ v i v o ~ a c u t e ~ h i p p o c a m p a l ~ s l i c e s . ~} A$, LTP was measured in regular spiking cell synapses after in vivo molecular replacement with Nrxn3 $\alpha$ WT SS4- or Nrxn3 $\alpha^{\text {A687T }}$ SS4 - . LTP was induced by a $4 \times 100 \mathrm{~Hz}$ stimuli for $1 \mathrm{~s}$ with 10 s intervals while in current-clamp mode at resting membrane potential. After induction, cells were held at $-70 \mathrm{mV}$ in voltage-clamp mode to monitor EPSCs. Representative traces (top) and summary graphs (bottom). Representative traces shown are EPSC during baseline (1) and 60 min after LTP induction (2). B, Presynaptic LTP at CA1-Burst spiking cell synapses is impaired following molecular presynaptic replacement with Nrxn3 $\alpha^{\text {A687T }}$ SS4 - . Representative traces (top) and summary graphs (bottom). C, Representative traces (left) and summary graph (right) for PPRs with 50 ms ISIs monitored at CA1-RS cell synapses. PPRs were collected immediately before LTP induction (pre) and 60 min after induction (post). D, Same as C but for CA1-BS cell synapses before and after LTP induction. Data shown are mean \pm SEM; mean values calculated from the number of cells analyzed. Numbers in bars represent the number of animals used for each experiment. Statistical significance was determined by one-way ANOVA, multiple comparisons. n.s., not significant, ${ }^{*} p<0.05,{ }^{* * * *} p<0.0001$, \#\#\#\# $<0.0001$.

burst spiking neurons exhibit distinct forms of long-term potentiation (LTP). Although LTP at CA1-RS and CA1-BS cell synapses are both dependent on NMDAR activity, LTP is expressed postsynaptically at CA1-RS synapses but LTP manifests presynaptically as an increase in release probability at BS synapses (Wozny et al., 2008). To induce LTP at CA1-subiculum synapses, we administered a $4 \times 100 \mathrm{~Hz}$ electrical stimulus to CA1 afferents for $1 \mathrm{~s}$ with $10 \mathrm{~s}$ intervals while in whole-cell current-clamp mode at resting membrane potential. Synaptic responses pre- and postLTP induction were acquired while holding the postsynaptic cell at $-70 \mathrm{mV}$ in whole-cell voltage-clamp mode. Molecular replacement with presynaptic Nrxn3 $\alpha^{\text {A687T }}$ SS4 - did not alter the magnitude of RS cell LTP, as all conditions exhibited a 150$160 \%$ potentiation (shRNA: mean $=1.628 \pm 0.04216, p=$ 0.9380; shRNA/WT SS4 $-1.605 \pm 0.05447, p=0.7723$; shRNA/ A687T SS4 $-1.675 \pm 0.04666, p=0.583$; Fig. $9 A$ ). In stark contrast, presynaptic molecular replacement with $\operatorname{Nrxn} 3 \alpha^{\mathrm{A} 687 \mathrm{~T}}$ SS4-, but not $\operatorname{Nrxn} 3 \alpha^{\text {WT }}$ SS4-, resulted in impaired LTP at CA1-BS cell synapses (shRNA $1.793 \pm 0.02224, n=11$; shRNA/WT SS4 $-1.802 \pm 0.02901, n=11$; shRNA/A687T SS4$0.995 \pm 0.02366, n=11$, shRNA vs shRNA/A687T SS4-: $p \leq$
0.0001; shRNA/WT SS4- vs shRNA/A687T SS4-: $p \leq 0.0001$; one-way ANOVA, multiple comparisons; Fig. 9B). The impairment of LTP specifically at CA1-burst spiking cell synapses was surprising, yet our functional and morphological characterization in primary culture and hippocampal slices strongly support the notion that presynaptic Nrxn3 $\alpha^{\text {A687T }}$ SS4 - enhances excitatory presynaptic efficacy. Thus, we hypothesized that the Nrxn3 $\alpha^{\text {A687T }}$ SS4 - mediated increase in basal presynaptic efficacy occluded presynaptic LTP at CA1-BS synapses.

To test whether the increased presynaptic release probability measured in Nrxn3 $\alpha^{\text {A687T }}$ SS4 - infected slices occluded presynaptic LTP at CA1-BS cell synapses, we measured PPRs $10 \mathrm{~min}$ before and $60 \mathrm{~min}$ after LTP induction for control (shRNA alone) and molecular replacement $\left(\operatorname{Nrxn} 3 \alpha^{\text {WT }}\right.$ SS4 - and Nrxn $3 \alpha^{\mathrm{A} 687 \mathrm{~T}}$ SS4-) conditions. Consistent with a postsynaptic locus of LTP at CA1-RS synapses that was not affected by Nrxn3 $\alpha^{\text {A687T }}$ SS4-, LTP induction at these synapses did not increase presynaptic release probabilities (shRNA, pre: $1.90 \pm 0.273$, post: $1.77 \pm$ $0.218, n=9 / 6, p=0.379$; shRNA/WT SS4 - , pre: $1.72 \pm 0.105$, post: $1.82 \pm 0.274, n=11 / 9, p=0.682$; shRNA/A687T SS4-, pre: $1.674 \pm 0.122$, post: $2.04 \pm 0.150, n=14 / 10, p=0.0289$; 
one-way ANOVA, multiple comparisons; Fig. 9C). Interestingly, we observed a small but statistically significant increase in the PPR for the Nrxn3 $\alpha^{\text {A687T }}$ SS4 - condition, which suggests that even though presynaptic release probability was decreased, we still observed robust LTP at CA1-RS synapses. This further supports the notion that LTP is expressed postsynaptically at these synapses. In contrast, at CA1-BS synapses, presynaptic release probability after LTP induction was significantly increased in control and Nrxn $3 \alpha{ }^{\text {WT }}$ SS4 - conditions, however, release probability was not further enhanced when $\operatorname{Nrxn} 3 \alpha^{\text {A687T }}$ SS4- was expressed in presynaptic CA1 neurons. (shRNA, pre: $2.183 \pm$ $0.1317, n=5$, post: $1.293 \pm 0.2964, n=3, p=0.0188$; shRNA/WT SS4 - pre: $1.852 \pm 0.1424, n=6$, post: $1.346 \pm$ $0.08974, n=5, p=0.0189$; shRNA/A687T SS4-, pre: $1.697 \pm$ 0.0996 , post: $1.736 \pm 0.09715, n=6, p=0.7965$; one-way ANOVA, multiple comparisons; Fig. 9D). Together, these data indicate that the basal Nrxn3 $\alpha^{\mathrm{A} 687 \mathrm{~T}} \mathrm{SS} 4-$ induced enhancement of presynaptic release probability at CA1-BS cell synapses occludes additional potentiation following LTP induction further suggesting that this linker region in Neurexin-3 $\alpha$ between LNS4 and EGF2 is important for regulating presynaptic efficacy and the manifestation of LTP at these synapses.

\section{Nrxn3 $\alpha^{\mathrm{A} 687 \mathrm{~T}}$ selectively enhances aggregation with the excitatory ligand, LRRTM2}

The presynaptic gain-of-function phenotype is restricted to the splice-site 4 lacking Nrxn3 $\alpha^{\mathrm{A} 687 \mathrm{~T}}$ isoform, suggesting that SS4dependent transsynaptic interactions are required. We posited that the A687T extracellular mutation might alter binding to ligands which exclusively localize to excitatory synapses and preferentially bind to SS4-lacking neurexins, such as neuroligin-1 (NL1) and leucine rich repeat transmembrane protein 2 LRRTM2 (Südhof, 2008; Ko et al., 2009; de Wit et al., 2009). NL1 and LRRTM2 have redundant roles in excitatory synapse formation; however, NL1 and LRRTM2 also have nonredundant roles at excitatory synapses. Whereas NL1 controls NMDAR synaptic strength, LRRTM2 controls AMPAR synaptic strength (SolerLlavina et al., 2011; Budreck et al., 2013; Wu et al., 2019). Intriguingly, similar to the phenotype observed following molecular replacement with $\operatorname{Nrxn} 3 \alpha^{\mathrm{A} 687 \mathrm{~T}} \mathrm{SS} 4-$, overexpression of postsynaptic LRRTM2 robustly increases presynaptic vGluT1 cluster size (de Wit et al., 2009). Moreover, Nrxn3 SS4- has previously been shown to be a functionally important interaction partner with LRRTM2 at excitatory synapses in hippocampus (Aoto et al., 2013). Therefore, interactions of $\alpha$-Nrxns with NL1 and LRRTM2 can encode for distinct aspects of synapse function.

To test whether A687T alters interactions with NL1-3 and LRRTM2 in trans, we used a HEK cell aggregation assay. In one population of HEK293T cells, NL1, NL2, NL3, or LRRTM2 were cotransfected with GFP while in a second population of HEK293T cells, SS4 isoforms of WT or A687T Nrxn3 $\alpha$ were cotransfected with mCherry. These two populations of cells were incubated in suspension before aggregation was quantified by the colocalization of GFP with mCherry (Fig. 10A). Cell aggregation in conditions where GFP or mCherry were expressed alone was not observed (Fig. 10). The A687T mutation did not alter aggregation with any neuroligin compared with WT $(p=0.1452$; Fig. $10 B, C, F-I)$. Unexpectedly, relative to $\operatorname{Nrxn} 3 \alpha^{\mathrm{WT}}$ conditions, we observed that Nrxn3 $\alpha^{\text {A687T }}$ SS4- induced a striking increase in aggregation with LRRTM2 (WT SS4-: $28 \pm 5.4 \%, n=3$; A687T SS4-: $45 \pm 1.7 \%, n=3, p=0.0136$; one-way ANOVA, multiple comparisons; Fig. 10E). Consistent with the idea that LRRTM2 only binds to SS4-lacking neurexins, we failed to observe aggre- gation when LRRTM2 was co-incubated with either WT or A687T Nrxn3 $\alpha$ SS4+ (Fig. 10D). Highlighting the importance of this unstudied linker region, our data suggest that at excitatory synapses, $\operatorname{Nrxn} 3 \alpha^{\mathrm{A} 687 \mathrm{~T}} \mathrm{SS} 4-$ preferentially interacts with LRRTM2, which changes synaptic signaling and enhances presynaptic function.

\section{Discussion}

Here, we identified a novel missense mutation in a neurexin- $3 \alpha$ compound heterozygous patient diagnosed with profound intellectual disability and epilepsy and systematically studied this mutation using molecular replacement in primary cultures and ex vivo acute slices. The mutation, located in a completely unstudied but evolutionarily conserved region, provided an avenue to directly probe the functional role of this extracellular sequence of $\operatorname{Nrxn} 3 \alpha$. Our results reveal that this sequence is a critical mediator in controlling trans interactions with postsynaptic ligands, presynaptic morphology, and release probability specifically at excitatory synapses. Perhaps it is not surprising that a mutation in Nrxn $3 \alpha$ results in a presynaptic phenotype, given that neurexins are essential presynaptic proteins, however, it is remarkable that the phenotypes reported here are not observed in the Nrxn3 $\alpha$ KO. Moreover, to the best of our knowledge, we identify the first manipulation of any neurexin that results in a potent but selective gain-of-function presynaptic phenotype at excitatory synapses. In addition to identifying a novel and unpredicted role for Nrxn $3 \alpha$ extracellular sequences, our results highlight the value of studying these naturally occurring missense mutations because they have significant potential to provide novel functional insight that might otherwise be obscured in KO models.

In primary culture, molecular replacement with $\operatorname{Nrxn} 3 \alpha^{\mathrm{A} 687 \mathrm{~T}}$ SS4 - but not Nrxn $3 \alpha^{\mathrm{A} 687 \mathrm{~T}} \mathrm{SS} 4+$ significantly increased vGluT1 cluster size independent of changes in excitatory synapse density or inhibitory synaptic morphology (Fig. $3 G, H$ ) and enhanced mEPSC frequency, increased evoked AMPAR- and NMDARmediated synaptic transmission (Fig. $5 F-I$ ). Additionally, our data suggest that the striking presynaptic gain-of-function phenotype could be explained by changes in two presynaptic properties: (1) increased presynaptic release probability (Fig. 5I), and (2) enlarged the size of the excitatory RRP (Fig. $7 B, C$ ). In acute hippocampal slices, we found that presynaptic molecular replacement with Nrxn3 $\alpha^{\text {A687T }}$ SS4 - at synapses formed by afferents from CA1 onto postsynaptic subicular RS and BS neurons significantly enhanced AMPAR-mediated synaptic strength and increased excitatory presynaptic release probability (Fig. 8D-G). Surprisingly, increased neurotransmitter release induced by Nrxn3 $\alpha^{\text {A687T }}$ SS4 - occluded presynaptic, but not postsynaptic, LTP at CA1-subiculum synapses (Fig. 9).

We used cell aggregation assays to gain mechanistic insight into how the A687T mutation alters Nrxn3 $\alpha$ function. We surprisingly found that the A687T mutation selectively enhanced trans interactions with LRRTM2 without altering interactions with any neuroligins (Fig. 10). LRRTM2 and NLs bind to the same surface in LNS6 (Siddiqui et al., 2010), which suggests that the mutation does not alter the direct binding properties of LNS6 but independently exerts its effect. How might enhanced $\operatorname{Nrxn} 3 \alpha$ binding with LRRTM2 affect synaptic transmission? Elegant single molecule tracking experiments demonstrated that NL1 is uniformly distributed at excitatory post-synapses, whereas LRRTM2 forms tight domains near the core of the postsynaptic density (Chamma et al., 2016). An attractive explanation, based on single molecule tracking and our results presented here, is that the A687T mutation in Nrxn3 $\alpha$ SS4- may cause the presynaptic 
A

Nrxn3a Aggregation Assay
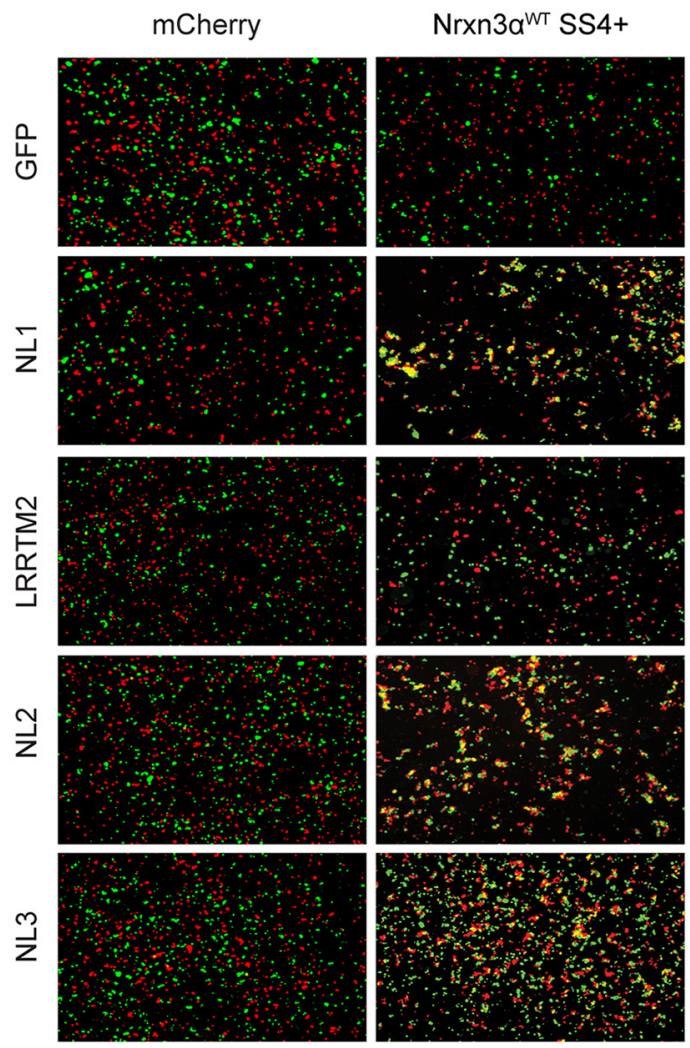

B

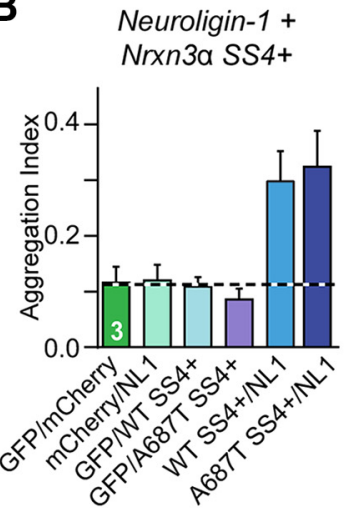

F

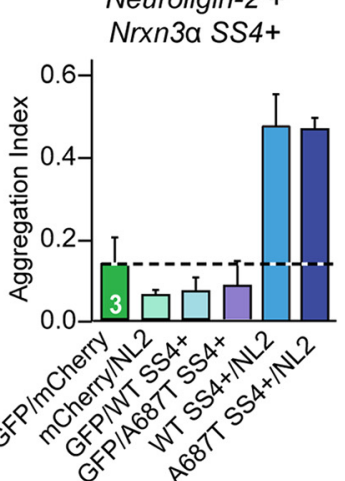

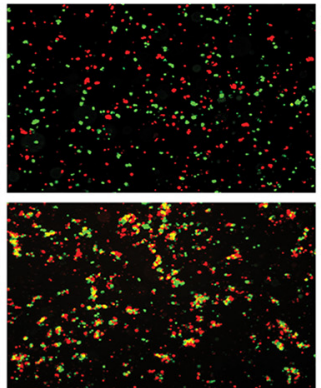

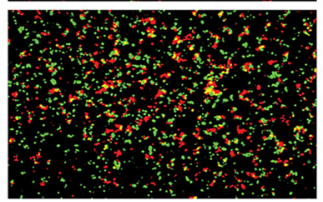

C

Neuroligin-1 + Nrxn3a SS4-

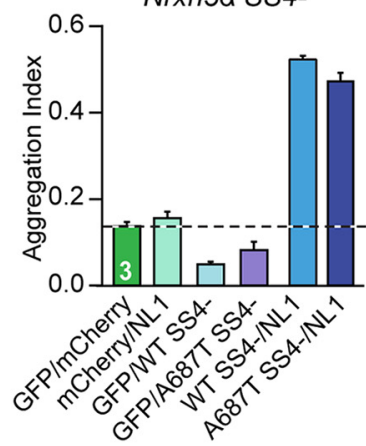

G

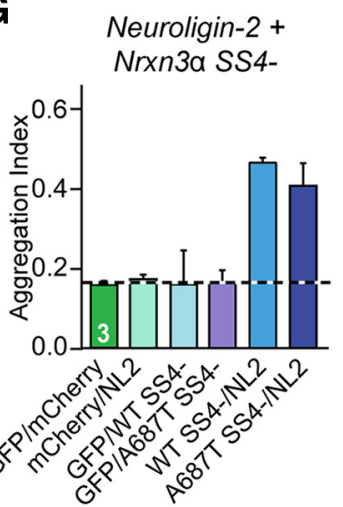

Nrxn3 $\alpha^{\text {A687T }}$ SS4+

Nrxn3awT SS4-
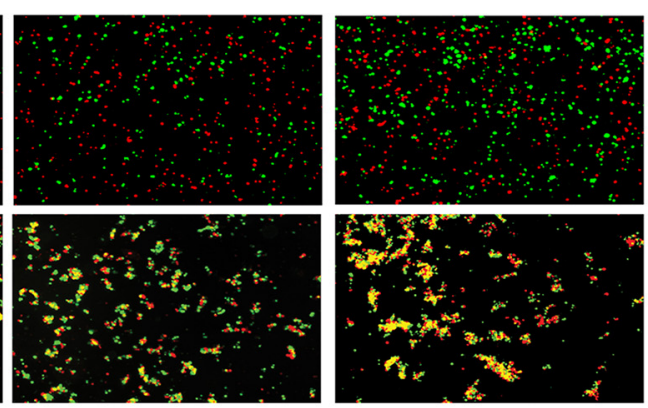

Nrxn3 $a^{\text {A687T }}$ SS4-
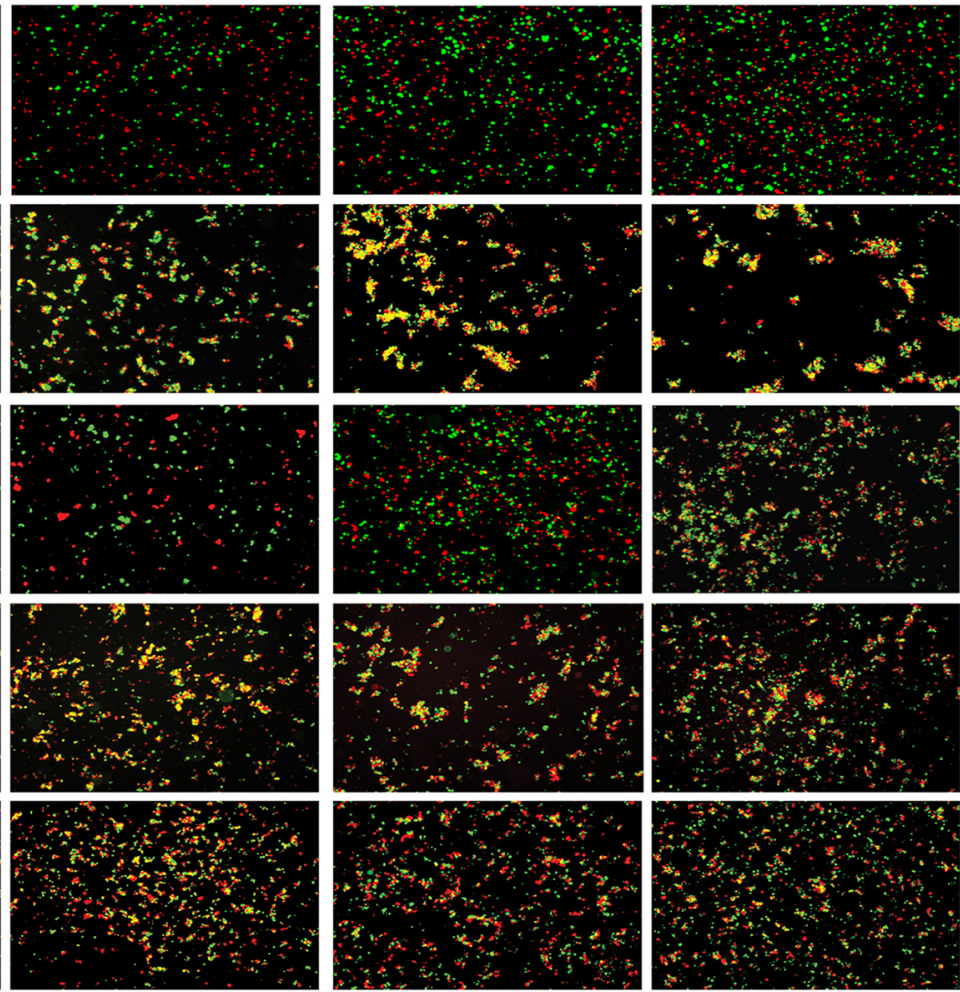

D

LRRTM2 + Nrxn3a SS4+

E

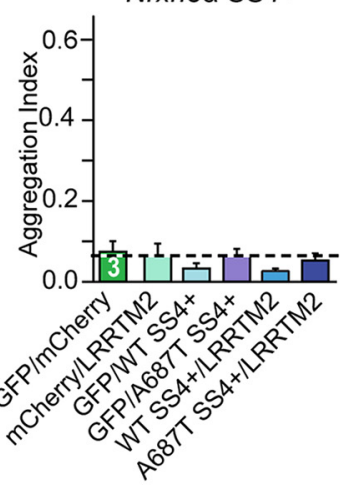

H
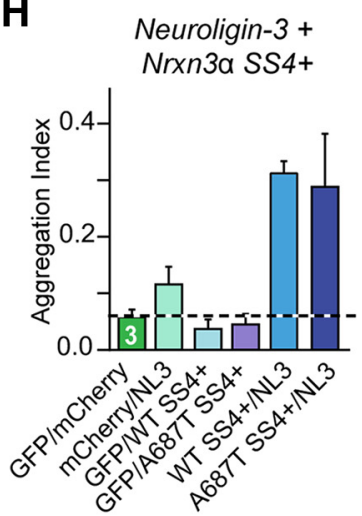

LRRTM2 +

Nrxn3a SS4-

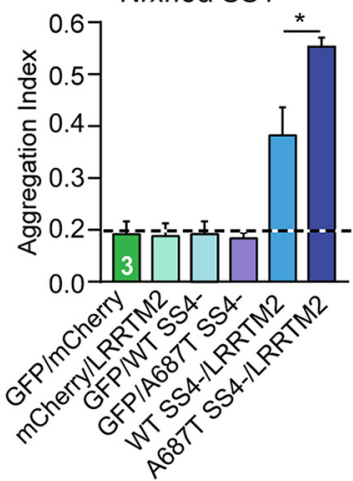

I

Neuroligin-3+ Nrxn3a SS4-

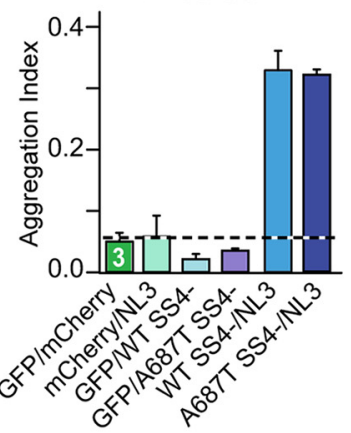

Figure 10. The A687T mutation selectively increases Nrxn3 $\alpha$ SS4 - binding to LRRTM2 but not to neuroligins. $A$, Representative images of the HEK cell aggregation assay. $\boldsymbol{B}-\mathbf{I}$, Summary graphs of quantification of HEK cell aggregation assays. One population of HEK cells were transfected with SS4 isoforms of Nrxn $3 \alpha{ }^{\text {WT }}$ or Nrxn $3 \alpha^{\text {A687T }}$ and co-incubated with HEK cells transfected with the neurexin ligands NL1 (B) and $(\boldsymbol{C}), \operatorname{LRRTM2}(\boldsymbol{D})$ and $(\boldsymbol{E}), \mathrm{NL2}(\boldsymbol{F})$ and $(\boldsymbol{G})$, or NL3 $(\boldsymbol{H})$ and $(\boldsymbol{I})$. The index of aggregation was calculated by measuring the number of colocalized cells (cells with red + green overlap) divided by the total number of red and green cells. Data shown are mean $\pm \mathrm{SEM}$; mean values are calculated from average of individual experiments analyzed. Numbers in bars represent the number of independent experiments. Statistical significance was determined by one-way ANOVA, multiple comparisons. ${ }^{*} p<0.05$. 
rearrangement of the molecular architecture. Under normal circumstances, Nrxn3 $\alpha^{\text {WT }}$ SS4- interacts with NL1 and LRRTM2, and is evenly distributed in the presynaptic bouton, however, the preferential interaction of $\mathrm{Nrxn} 3 \alpha^{\mathrm{A} 687 \mathrm{~T}} \mathrm{SS} 4-$ with LRRTM2 may cause a redistribution of $\mathrm{Nrxn} 3 \alpha^{\mathrm{A} 687 \mathrm{~T}} \mathrm{SS} 4-$ and its intracellular signaling components more centrally in the AZ, ultimately leading to the observed presynaptic gain-of-function phenotype. Unfortunately, how Nrxn3 $\alpha^{\mathrm{A} 687 \mathrm{~T}} \mathrm{SS} 4-$ functions to increase excitatory RRP size and presynaptic release probability via intracellular signaling is unknown because our understanding of the intracellular signaling downstream of neurexins is incomplete. Moreover, the answer to the fundamental question: "do individual neurexins engage distinct or redundant presynaptic intracellular signaling pathways?" is unknown. These data emphasize the growing importance of the transsynaptic adhesion code, i.e., how precise cell-type-specific neurexin isoforms govern interactions at the synapse to promote neurotransmission, and suggest that the presynaptic localization of $\alpha$-neurexin interactions is profoundly important to function.

Given that $\operatorname{Nrxn} 3 \alpha$ is highly expressed in many GABAergic interneuron subtypes in hippocampus, it is surprising that the A687T mutation did not affect inhibitory synapse morphology or function (Ullrich et al., 1995; Nguyen et al., 2016; Lukacsovich et al., 2019). Indeed, genetic deletion of $\operatorname{Nrxn} 3 \alpha$ significantly impaired presynaptic release at inhibitory synapses in olfactory bulb neurons and deletion of both $\operatorname{Nrxn} 3 \alpha$ and $\operatorname{Nrxn} 3 \beta$ in anterior cingulate cortex impaired neurotransmitter release from somatostatin interneurons (Aoto et al., 2015; Keum et al., 2018). A technical limitation to this study is the use of $\operatorname{Nrxn} 3 \alpha$ replacement cDNAs, which are not subject to alternative splicing. Here, we manipulated the inclusion of the SS4 exon without testing the impact of the other splice sites, which may be crucial for GABAergic interneuron function (Fuccillo et al., 2015; Nguyen et al., 2016; Lukacsovich et al., 2019). To gain a more comprehensive understanding, it will be critical to study $\operatorname{Nrxn} 3 \alpha^{\mathrm{A} 687 \mathrm{~T}}$ and other $\alpha$-Nrxn missense mutations in an environment where splicing is controlled by endogenous mechanisms unique to each cell type.

Our study represents the first systematic functional interrogation of a neurexin- $3 \alpha$ human mutation. Interestingly, a recent study identified a naturally occurring extracellular mutation in neurexin-3 $\alpha$ (R498W) in a $129 \mathrm{~S} 1 /$ SvlmJ mouse strain (Keum et al., 2018). The R498W mutation is located in LNS3, which is upstream of the mutation studied here (Fig. 1A; linker between EGF2 and LNS4). Keum et al. (2018) demonstrated that the R498W mutation specifically enhanced fear empathy in 129S1/ SvlmJ mice relative to other commonly used mouse lines that contain an arginine at position 498 of Nrxn3 $\alpha$. They posited that the mutation in $\operatorname{Nrxn} 3 \alpha$ impaired presynaptic release selectively at synapses made by somatostatin interneurons in anterior cingulate cortex (ACC), thus, in ACC Nrxn3 $\alpha$ extracellular sequences appear to necessary for the release of inhibitory neurotransmitter at somatostatin synapses, however, the mutation itself was not directly functionally assayed at those synapses. By contrast, here we demonstrate that a completely different extracellular region of $\operatorname{Nrxn} 3 \alpha$ is responsible for controlling presynaptic release and the size of the RRP at excitatory synapses in hippocampus. Together, these data suggest that different extracellular sequences specific for $\alpha$ neurexins may have the capacity to govern synaptic transmission in a brain-region, cell-type-, and synapse-specific manner.

To the best of our knowledge, our findings represent the first interrogation of a human neurexin mutation and the first reported gain-of-function manipulation of any neurexin, which significantly strengthens our understanding of the synapsespecific roles of $\alpha$-Nrxn extracellular sequences. The discovery of the Nrxn $3 \alpha^{\mathrm{A} 687 \mathrm{~T}}$ phenotype, unpredicted based on neurexin KO models, highlights the utility of studying patient mutations in genes that encode neurexins, increasing our knowledge of neurexin biology and providing insight into how these mutations alter synapse function that underlie neuropsychiatric and neurodevelopmental disorders.

\section{References}

Anderson GR, Aoto J, Tabuchi K, Földy C, Covy J, Yee AX, Wu D, Lee SJ, Chen L, Malenka RC, Südhof TC (2015) $\beta$-neurexins control neural circuits by regulating synaptic endocannabinoid signaling. Cell 162:593606.

Aoto J, Martinelli DC, Malenka RC, Tabuchi K, Südhof TC (2013) Presynaptic neurexin-3 alternative splicing trans-synaptically controls postsynaptic AMPA receptor trafficking. Cell 154:75-88.

Aoto J, Földy C, Ilcus SM, Tabuchi K, Südhof TC (2015) Distinct circuitdependent functions of presynaptic neurexin-3 at GABAergic and glutamatergic synapses. Nat Neurosci 18:997-1007.

Bacaj T, Wu D, Burré J, Malenka RC, Liu X, Südhof TC (2015) Synaptotagmin-1 and -7 are redundantly essential for maintaining the capacity of the readily-releasable pool of synaptic vesicles. PLoS Biol 13: e1002267.

Benson DL, Cohen PA (1996) Activity-independent segregation of excitatory and inhibitory synaptic terminals in cultured hippocampal neurons. J Neurosci 16:6424-6432.

Benson DL, Watkins FH, Steward O, Banker G (1994) Characterization of GABAergic neurons in hippocampal cell cultures. J Neurocytol 23:279295.

Budreck EC, Kwon O-B, Jung JH, Baudouin S, Thommen A, Kim H-S, Fukazawa Y, Harada H, Tabuchi K, Shigemoto R, Scheiffele P, Kim J-H (2013) Neuroligin-1 controls synaptic abundance of NMDA-type glutamate receptors through extracellular coupling. Proc Natl Acad Sci U S A 110:725-730.

Chamma I, Letellier M, Butler C, Tessier B, Lim KH, Gauthereau I, Choquet D, Sibarita JB, Park S, Sainlos M, Thoumine O (2016) Mapping the dynamics and nanoscale organization of synaptic adhesion proteins using monomeric streptavidin. Nat Commun 7:10773.

Chanaday NL, Kavalali ET (2018) Presynaptic origins of distinct modes of neurotransmitter release. Curr Opin Neurobiol 51:119-126.

Chen LY, Jiang M, Zhang B, Gokce O, Südhof TC (2017) Conditional deletion of all neurexins defines diversity of essential synaptic organizer functions for neurexins. Neuron 94:611-625.e4.

Crawford DC, Ramirez DM, Trauterman B, Monteggia LM, Kavalali ET (2017) Selective molecular impairment of spontaneous neurotransmission modulates synaptic efficacy. Nat Commun 8:14436.

Dai J, Aoto J, Südhof TC (2019) Alternative splicing of presynaptic neurexins differentially controls postsynaptic NMDA and AMPA receptor responses. Neuron 102:993-1008.e5.

de Wit J, Sylwestrak E, O'Sullivan ML, Otto S, Tiglio K, Savas JN, Yates JR 3rd, Comoletti D, Taylor P, Ghosh A (2009) LRRTM2 interacts with Neurexin 1 and regulates excitatory synapse formation. Neuron 64:799-806.

Etherton MR, Blaiss CA, Powell CM, Südhof TC (2009) Mouse neurexinlalpha deletion causes correlated electrophysiological and behavioral changes consistent with cognitive impairments. Proc Natl Acad Sci U S A 106:17998-18003.

Fuccillo MV, Földy C, Gökce Ö, Rothwell PE, Sun GL, Malenka RC, Südhof TC (2015) Single-cell mRNA profiling reveals cell-type-specific expression of neurexin isoforms. Neuron 87:326-340.

Kaeser PS, Regehr WG (2017) The readily releasable pool of synaptic vesicles. Curr Opin Neurobiol 43:63-70.

Keum S, Kim A, Shin JJ, Kim JH, Park J, Shin HS (2018) A missense variant at the Nrxn3 locus enhances empathy fear in the mouse. Neuron 98:588 601.e5.

Ko J, Fuccillo MV, Malenka RC, Südhof TC (2009) LRRTM2 functions as a neurexin ligand in promoting excitatory synapse formation. Neuron 64: 791-798.

Koehnke J, Katsamba PS, Ahlsen G, Bahna F, Vendome J, Honig B, Shapiro L, Jin X (2010) Splice form dependence of $\beta$-neurexin/neuroligin binding interactions. Neuron 67:61-74. 
Liu Q, Sinnen BL, Boxer EE, Schneider MW, Grybko MJ, Buchta WC, Gibson ES, Wysoczynski CL, Ford CP, Gottschalk A, Aoto J, Tucker CL, Kennedy MJ (2019) A photoactivatable botulinum neurotoxin for inducible control of neurotransmission. Neuron 101:863-875.e6.

Lukacsovich D, Winterer J, Que L, Luo W, Lukacsovich T, Földy C (2019) Single-cell RNA-Seq reveals developmental origins and ontogenetic stability of neurexin alternative splicing profiles. Cell Rep 27:3752-3759.e4.

Miller MT, Mileni M, Comoletti D, Stevens RC, Harel M, Taylor P (2011) The crystal structure of the $\alpha$-neurexin-1 extracellular region reveals a hinge point for mediating synaptic adhesion and function. Structure 19:767-778.

Missler M, Zhang W, Rohlmann A, Kattenstroth G, Hammer RE, Gottmann K, Südhof TC (2003) Alpha-neurexins couple $\mathrm{Ca}^{2+}$ channels to synaptic vesicle exocytosis. Nature 423:939-948.

Nguyen TM, Schreiner D, Xiao L, Traunmüller L, Bornmann C, Scheiffele P (2016) An alternative splicing switch shapes neurexin repertoires in principal neurons versus interneurons in the mouse hippocampus. eLife 5:e22757.

Pak C, Danko T, Zhang Y, Aoto J, Anderson G, Maxeiner S, Yi F, Wernig M, Südhof TC (2015) Human neuropsychiatric disease modeling using conditional deletion reveals synaptic transmission defects caused by heterozygous mutations in NRXN1. Cell Stem Cell 17:316-328.

Ramirez DM, Kavalali ET (2011) Differential regulation of spontaneous and evoked neurotransmitter release at central synapses. Curr Opin Neurobiol 21:275-282.

Reissner C, Runkel F, Missler M (2013) Neurexins. Genome Biol 14:213.

Siddiqui TJ, Pancaroglu R, Kang Y, Rooyakkers A, Craig AM (2010)
LRRTMs and neuroligins bind neurexins with a differential code to cooperate in glutamate synapse development. J Neurosci 30:7495-7506.

Soler-Llavina GJ, Fuccillo MV, Ko J, Südhof TC, Malenka RC (2011) The neurexin ligands, neuroligins and leucine-rich repeat transmembrane proteins, perform convergent and divergent synaptic functions in vivo. Proc Natl Acad Sci U S A 108:16502-16509.

Südhof TC (2008) Neuroligins and neurexins link synaptic function to cognitive disease. Nature 455:903-911.

Südhof TC (2017) Synaptic neurexin complexes: a molecular code for the logic of neural circuits. Cell 171:745-769.

Ullrich B, Ushkaryov YA, Südhof TC (1995) Cartography of neurexins: more than 1000 isoforms generated by alternative splicing and expressed in distinct subsets of neurons. Neuron 14:497-507.

Wozny C, Maier N, Schmitz D, Behr J (2008) Two different forms of longterm potentiation at CA1-subiculum synapses. J Physiol 586:2725-2734.

Wu X, Morishita WK, Riley AM, Hale WD, Südhof TC, Malenka RC (2019) Neuroligin-1 signaling controls LTP and NMDA receptors by distinct molecular pathways. Neuron 102:621-635.e3.

Yan J, Noltner K, Feng J, Li W, Schroer R, Skinner C, Zeng W, Schwartz CE, Sommer SS (2008) Neurexin $1 \alpha$ structural variants associated with autism. Neurosci Lett 438:368-370.

Zhang W, Rohlmann A, Sargsyan V, Aramuni G, Hammer RE, Südhof TC, Missler M (2005) Extracellular domains of -neurexins participate in regulating synaptic transmission by selectively affecting $\mathrm{N}$ - and P/Q-type $\mathrm{Ca}^{2+}$ channels. J Neurosci 25:4330-4342. 